\title{
Enquête Ethnobotanique Auprès Des Tradipraticiens De Santé Des Régions De Niamey Et Tillabéri Au Niger: Données 2012-2017
}

\author{
Mamadou Aïssa Jazy \\ Laboratoire de Botanique- Pharmacognosie, Faculté des Sciences de la \\ Santé, Université Abdou Moumouni, Niamey, Niger \\ Saley Karim \\ Boubé Morou \\ Laboratoire de Biologie, Faculté des Sciences et Techniques, \\ Université Dan Dicko Dankoulodo, Maradi, Niger

\section{Rokia Sanogo} \\ DER des Sciences Pharmaceutiques, Faculté de Pharmacie, \\ Université de Bamako, Mali \\ Département de Médecine Traditionnelle, Institut National de Recherche en \\ Santé Publique, Ministère de la Santé Publique, Bamako, Mali

\section{Saadou Mahamane} \\ Laboratoire de Biologie, Faculté des Sciences et Techniques, \\ Université Dan Dicko Dankoulodo, Maradi, Niger
}

Doi: 10.19044/esj.2017.v13n33p276 URL:http://dx.doi.org/10.19044/esj.2017.v13n33p276

\begin{abstract}
Introduction: In Niger, people employ the services of traditional health practitioners who use medicinal recipes and plants for primary health care. Scope: Identify medicinal plants for primary health care. Methods: An ethnobotanical survey was carried out in two regions of Niger, Tillabéri and Niamey, according to a questionnaire. Results: This study assessed the transmission of knowledge, understood the concept of dosage in traditional medicine, and established a repertoire of recipes and plants of the traditional pharmacopoeia. A total of 913 therapeutic recipes for 226 species belonging to 77 families were collected from 168 traditional practitioners. The species most frequently encountered are: Sclerocarya birrea, Acacia nilotica, Guiera senegalensis, Annona senegalensis, Combretum micranthum, Balanites aegyptiaca, Pennisetum americanum, Piliostigma reticulatum, Cassia sieberiana, Ziziphus mauritiana, Azadirachta indica, Cassia occidentalis, Anogeissus leiocarpus, Combretum glutinosum, Khaya senegalensis, Momordica balsamina, Tephrosia lupunifolia, Prosopis africana, Alysicarpus ovalifolius, Bauhinia rufescens. The most common among them
\end{abstract}


is the Combretaceae (10.68\%), followed by Fabaceae (9.67\%), Caesalpiniaceae (8.49\%), Mimosaceae (7.82\%), and Anacardiaceae (5, $55 \%$ ). Among these plants, 60 belonging to 31 families, including 6 new families, were recorded. Several pathologies were treated. The most frequent of them are digestive disorders, hemorrhoids. Conclusion: These plants will be subjected to experimental studies before the development of new phytomedicines.

Keywords: Traditional practitioners, Ethnobotany, Niamey, Tillaberi, Niger

\section{Résumé}

Introduction: Au Niger, les populations font un grand recours aux tradipraticiens de santé qui utilisent des recettes et des plantes médicinales pour les soins de santé primaires. Objectif: Recenser les plantes médicinales pour les soins de santé primaires. Méthodologie: Une enquête ethnobotanique effectuée dans deux régions du Niger: Tillabéri et Niamey, selon un questionnaire. Résultats: L'étude a permis d'apprécier la transmission des connaissances, d'appréhender la notion de posologie en médecine traditionnelle, et d'établir un répertoire des recettes et des plantes de la pharmacopée traditionnelle. Au total 913 recettes thérapeutiques relatives à 226 espèces appartenant à 77 familles sont recueillies auprès de 168 tradipraticiens. Les espèces les plus fréquemment rencontrées sont: Sclerocarya birrea, Acacia nilotica, Guiera senegalensis, Annona senegalensis, Combretum micranthum, Balanites aegyptiaca, Pennisetum americanum, Piliostigma reticulatum, Cassia sieberiana, Ziziphus mauritiana, Azadirachta indica, Cassia occidentalis, Anogeissus leiocarpus, Combretum glutinosum, Khaya senegalensis, Momordica balsamina, Tephrosia lupunifolia, Prosopis africana, Alysicarpus ovalifolius, Bauhinia rufescens. La famille la plus représentée est celle des Combretaceae $(10,68 \%)$, suivie des Fabaceae $(9,67 \%)$, des Caesalpiniaceae $(8,49 \%)$, des Mimosaceae (7,82\%), puis des Anacardiaceae (5,55\%). Parmi ces plantes, 60 appartenant à 31 familles dont 6 familles nouvelles ont été répertoriées. Plusieurs pathologies sont traitées, les plus fréquentes sont les troubles digestifs, les hémorroïdes. Conclusion: Ces plantes seront soumises à des études expérimentales avant la mise au point de nouveaux phytomédicaments.

Mots-clés: Tradipraticiens, Ethnobotanique, Niamey, Tillabéri, Niger

\section{Introduction}

En Afrique subsaharienne notamment au Niger, la population aussi bien urbaine que rurale reste liée à la pharmacopée traditionnelle pour des raisons socioculturelles, mais aussi par contrainte due à l'accessibilité limitée 
des médicaments issus des pays développés (Schmelzer et al., 2008). Ceci est dû à la faiblesse des moyens financiers provoquée par la paupérisation des populations et l'insuffisance de la couverture sanitaire de l'Etat.

Les besoins en santé augmentent du fait de la démographie galopante malgré la priorité accordée au secteur. Au Niger la population est estimée à 17129076 en 2012, le taux d'accroissement est passé de 3,3\% au cours de la période 1998-2001, à 3,9\% pour la période 2001- 2012 (INS, 2012).

Il y a un regain d'intérêt pour la médecine et la pharmacopée traditionnelles. En Afrique noire, 70 à $80 \%$ de la couverture sanitaire est assurée par la médecine et la pharmacopée traditionnelles (Aké-Assi, 2006; Sofowora, 1996).

Plusieurs organismes internationaux reconnaissent à la médecine et la pharmacopée traditionnelles une utilité certaine et jouent un rôle déterminant dans la recherche sur la pharmacopée africaine (OUA, ONUDI, ACCT, OOAS, OMS).

En Afrique, le savoir socio-culturel, notamment concernant les plantes médicinales est détenu par les personnes généralement âgées qui transmettent rarement leurs connaissances. Leur disparition correspond à une importante perte d'informations profitables. Il est donc nécessaire de réaliser des études visant à documenter et sauvegarder les connaissances sur la pharmacopée traditionnelle. Notre étude s'inscrit dans ce cadre et consiste en une enquête ethnobotanique auprès des tradipraticiens de santé (TPS) dans les régions de Niamey et Tillabéri pour répertorier quelques plantes médicinales et leurs usages en thérapeutique traditionnelle.

\section{Méthodologie}

\section{Echantillonnage}

Il s'agit d'une enquête ethnobotanique, étude prospective transversale, à visée descriptive, ruéalisée entre Mars 2012 et Janvier 2017. L'enquête a été faite dans la zone ouest du pays, précisément dans les régions administratives de Niamey et Tillabéri, couvrant une superficie de $25000 \mathrm{~km}^{2}$ avec une population estimée à 3726463 habitants, dont 1 011277 à Niamey et 2715186 à Tillabéri (INS, 2012). Cette zone d'étude se trouve au sein de la partie ouest du pays qui concentre près des $2 / 3$ de ses ressources forestières.

Le choix de la région de Niamey est basé sur la forte concentration de la population urbaine et rurale toutes professions confondues dans la capitale, et celle de Tillabéri par proximité. Les départements et villages enquêtés ont été sélectionnés sur la base de leur accessibilité. 


\section{Méthode}

\section{Collecte des données}

Les données ont été collectées sur des fiches conçues à cet effet auprès de tous les tradipraticiens installés dans les deux régions et qui acceptent de participer à l'enquête, en décrivant leurs différentes recettes à base de plantes.

Outre les informations sur les TPS, sont notés les renseignements sur les plantes (nom vernaculaire, commun en français si possible, binôme latin, nom de famille, organes utilisés), et sur les recettes médicamenteuses (autres produits naturels entrant dans la composition du médicament, mode de préparation du médicament, durée de conservation, maladies soignées, quantité par prise, nombre de prises, moment des prises, durée du traitement, mode d'administration du médicament).

Les possibles erreurs (lapsus, oubli) lors de l'interrogatoire sont minimisées en posant la même question sur une recette de plusieurs manières afin de s'assurer que le TPS donne la même réponse.

\section{Analyse au laboratoire} répertoriées:

Identifications botaniques des plantes impliquées dans les recettes

La liste floristique est établie après détermination des espèces végétales des échantillons obtenus grâce aux documents de référence notamment les différentes Flores d'Afrique de l'Ouest (Berhaut, 1967; Akoègninou, 2006; Peyre De Fabregues, 1979; Saadou, 1998), et vérification au Laboratoire de Botanique du Département de Biologie à la Faculté des Sciences et Techniques de l'Université Dan Dicko Dankoulodo de Maradi. Un échantillon de référence est déposé audit laboratoire.

La recherche documentaire a permis de comparer la liste des espèces recensées et leurs usages thérapeutiques à celles des plantes déjà inventoriées comme médicinales dans des travaux antérieurs publiés notamment les enquêtes ethnobotaniques (Adjanohoun, 1980; Saadou, 1993).

\section{Difficultés rencontrés et limites de l'étude}

Nous avons souvent effectué des visites infructueuses car en général les TPS, même s'ils acceptent l'interview, ne donnent pas facilement leurs recettes. Plusieurs entretiens sont nécessaires, ce qui nous a pris beaucoup plus de temps que prévu.

Le refus de participer à l'enquête de certains tradipraticiens, surtout à Niamey, et l'inaccessibilité de certains endroits de la zone d'étude sont des limites à notre enquête. 


\section{Résultats}

\section{Données relatives aux Tradipraticiens}

Sur les 189 TPS contactés, 21 n'ont pas accepté de participer à l'enquête, soit un taux de refus de $11,1 \%$, variant de façon significative de $5,1 \%(8 / 156)$ à Tillabéri à 39,4\% (13/33) à Niamey.

Les principaux motifs sont : le manque de temps, la perte de temps, l'activité est lucrative, à ne pas exposer au risque de "piratage » par autrui (manque de confiance). Les principales caractéristiques sociodémographiques des 168 TPS enquêtés figurent dans le Tableau I.

Les personnes de notre enquête toutes musulmanes ont une durée d'exercice variant entre 2 et 75 ans ( 3 et 40 ans à Niamey, 2 et 75 ans à Tillabéri) avec une moyenne de 25,76 ans (Tableau I).

Tableau I. Principales données sociodémographiques des TPS enquêtés

\begin{tabular}{|c|c|c|c|c|c|c|}
\hline \multirow[b]{2}{*}{ Variable } & \multicolumn{2}{|c|}{ NIAMEY } & \multicolumn{2}{|c|}{ TILLABERI } & \multicolumn{2}{|c|}{ TOTAL } \\
\hline & Effectif & $\%$ & Effectif & $\%$ & Effectif & $\%$ \\
\hline \multicolumn{7}{|c|}{ Sexe } \\
\hline Masculin & 16 & 80 & 128 & 86,5 & 144 & 85,7 \\
\hline Féminin & 4 & 20 & 20 & 13,5 & 24 & 14,3 \\
\hline \multicolumn{7}{|c|}{ Age } \\
\hline$\leq 25$ ans & 0 & 0 & 9 & 6,1 & 9 & 5,4 \\
\hline$>25$ à $\leq 50$ ans & 10 & 50 & 56 & 37,84 & 66 & 39,3 \\
\hline$>50$ à $\leq 75$ ans & 10 & 50 & 76 & 51,35 & 86 & 51,2 \\
\hline$>75$ ans & 0 & 0 & 7 & 4,7 & 7 & 4,1 \\
\hline \multicolumn{7}{|c|}{ Origine des connaissances de TPS } \\
\hline Famille & 16 & 80 & 123 & 83,1 & 139 & 82,7 \\
\hline $\mathrm{F}+$ hors famille & 3 & 15 & 4 & 2,7 & 7 & 4,2 \\
\hline Hors famille & 1 & 5 & 21 & 14,2 & 22 & 13,1 \\
\hline \multicolumn{7}{|c|}{ Durée d'exercice } \\
\hline 1 à 10 ans & 3 & 15 & 30 & 20,3 & 33 & 19,64 \\
\hline$>10$ ans & 12 & 60 & 90 & 60,8 & 102 & 60,7 \\
\hline $\begin{array}{c}\text { Ne se souviens } \\
\text { pas }\end{array}$ & 5 & 25 & 28 & 18,9 & 33 & 19,64 \\
\hline \multicolumn{7}{|c|}{ Professions parallèles } \\
\hline TPS seulement & 17 & 85 & 49 & 33,11 & 66 & 39,3 \\
\hline Agriculteur & 2 & 10 & 70 & 47,3 & 72 & 42,86 \\
\hline $\begin{array}{c}\text { Autre occupation } \\
(*)\end{array}$ & 1 & 5 & 29 & 19,59 & 30 & 17,86 \\
\hline
\end{tabular}

(*) : Commerçant, matrone, éleveur, tailleur, forgeron, tresseuse, enseignant, féticheur, cuisinier.

\section{Recettes médicinales}

\section{Recettes et TPS}

$\mathrm{Au}$ total 913 recettes thérapeutiques relatives à 226 espèces appartenant à 76 familles sont recueillies auprès des 168 TPS qui font le diagnostic et procèdent au traitement des maladies décelées à l'aide des médicaments traditionnels qu'ils préparent. 
La prise en compte de dose d'administration, nombre de doses quotidiennes, moment d'administration, durée du traitement est variable d'une personne enquêtée à une autre. Elle est inconstante chez 149 TPS $(88,7 \%)$. Seulement 12 TPS ont donné une posologie précise pour toutes leurs recettes et 7 n'en ont pas fourni.

\section{Recettes, région, genre, plantes et produits associés}

A Tillabéri $796(87,19 \%)$ recettes ont été obtenues dont 117 auprès des femmes et 679 chez les hommes, contre $117(12,81 \%)$ à Niamey où 24 proviennent de femmes et 93 sont données par des hommes. (Tableau II)

Il y a en moyenne 5,43 recettes par personne enquêtée. Le nombre de recettes va de 1 à 22 avec une moyenne de 5,85 chez les personnes enquêtées de Niamey et de 1 à 26 chez celles de Tillabéri en moyenne 5,37 recettes par tradipraticien. Les recettes totales sont constituées en moyenne de 1,3 plantes. La majorité des recettes ( 741 soit $81,16 \%$ ) compte une seule espèce (81\% des recettes de Tillabéri, 82\% de celles de Niamey), $108(11,82 \%)$ en contiennent 2 (95 à Tillabéri soit 11,94\%, 13 à Niamey, soit 1,63\%). Seulement 19 recettes sur 913 (2\%) renferment 4 plantes ou plus. Le nombre maximal de plantes par recette est de 7 (5 à Niamey, 7 à Tillabéri).

Les 913 formules de préparation de médicaments emploient 1089 organismes végétaux entiers ou organes de plantes. Les parties utilisées sont le plus souvent l'appareil végétatif seul (828), la plante entière (230), l'appareil floral et/ou les fruits (100), mais aussi l'appareil végétatif et les fleurs ou fruits (25), rarement les sécrétions (6). Les plantes ou parties de plantes sont principalement utilisées seules lors de la préparation des médicaments traditionnels recensés dans l'ensemble (91,89\%), en association avec des produits minéraux $(5,15 \%)$, des produits végétaux $(1,1 \%)$, des produits animaux $(1,53 \%)$ ou simultanément plusieurs types de produits $(0,33 \%)$. La substance la plus employée dans l'association est le natron, suivie du sucre, du lait, du beurre animal à Tillabéri, et uniquement le sucre à Niamey.

Tableau II. Recettes selon la région, le genre, le nombre de plantes et les parties utilisées

\begin{tabular}{|c|c|c|c|c|c|c|}
\hline \multirow{2}{*}{ Variable } & \multicolumn{2}{|c|}{ Niamey } & \multicolumn{2}{c|}{ Tillabéri } & \multicolumn{2}{c|}{ Total } \\
\cline { 2 - 7 } & Effectif & $\%$ & Effectif & $\%$ & Effectif & $\%$ \\
\hline Sexe & & & & & & \\
\hline Hommes & 93 & 79,5 & 679 & 85,3 & 772 & 87,2 \\
\hline Femmes & 24 & 20,5 & 117 & 14,7 & 141 & 12,8 \\
\hline Total & 117 & 100 & 796 & 100 & 913 & 100 \\
\hline \multicolumn{7}{|c|}{ Nombre de plantes } \\
\hline 1 & 96 & 82 & 645 & 81 & 741 & 81,2 \\
\hline 2 & 13 & 11,2 & 95 & 11,9 & 108 & 11,8 \\
\hline 3 & 4 & 3,4 & 41 & 5,2 & 45 & 4,9 \\
\hline 4 ou plus & 4 & 3,4 & 15 & 1,9 & 19 & 2,1 \\
\hline Total & 117 & 100 & 796 & 100 & 913 & 100 \\
\hline
\end{tabular}




\begin{tabular}{|c|c|c|c|c|c|c|}
\hline \multicolumn{7}{|c|}{ Partie de la plante } \\
\hline $\begin{array}{c}\text { Appareil } \\
\text { végétatif }\end{array}$ & 89 & 58,9 & 737 & 71,2 & 828 & 69,7 \\
\hline $\begin{array}{c}\text { Appareil } \\
\text { floral/ fruits }\end{array}$ & 13 & 8 & 91 & 8,5 & 100 & 8,4 \\
\hline $\begin{array}{c}\text { Appareil } \\
\text { végétatif+ } \\
\text { Appareil } \\
\text { floral/ fruits }\end{array}$ & 7 & 4 & 21 & 1,8 & 25 & 2,1 \\
\hline Gommes/latex & 0 & 0 & 6 & 0,6 & 6 & 0,5 \\
\hline Plante entière & 42 & 29,1 & 183 & 17,9 & 230 & 19,3 \\
\hline Total & 151 & 100 & 1038 & 100 & 1189 & 100 \\
\hline \multicolumn{2}{|c|}{} & Produits associés & & & \\
\hline $\begin{array}{c}\text { Produits } \\
\text { minéraux }\end{array}$ & 5 & 4,3 & 42 & 5,3 & 47 & 5,2 \\
\hline $\begin{array}{c}\text { Produits } \\
\text { animaux }\end{array}$ & 0 & 0 & 14 & 1,8 & & 1,5 \\
\hline $\begin{array}{c}\text { Divers } \\
\text { produits }\end{array}$ & 0 & 0 & 13 & 1,6 & 13 & 1,4 \\
\hline Aucun & 112 & 95,7 & 727 & 91,3 & 839 & 91,9 \\
\hline Total & 117 & 100 & 796 & 100,00 & 913 & 100,00 \\
\hline
\end{tabular}

\section{Opérations, mode d'administration et durée de conservation des médicaments traditionnels}

Lors de la préparation des médicaments traditionnels plus du tiers des recettes dans les deux régions utilisent la décoction et la pulvérisation comme opérations.

Le mode d'emploi des médicaments est le plus souvent l'absorption (plus de 70\% de l'échantillon) suivie de l'application avec plus du quart des recettes. Certains médicaments sont destinés simultanément à la voie orale et à l'application externe $(5,98 \%$ des recettes de Niamey, 3,39 pour Tillabéri et $3,72 \%$ dans l'ensemble).

La durée de conservation est déterminée pour plus de la moitié des préparations médicamenteuses totales $(57,91 \%)$, et celles de Tillabéri (60,92\%), alors qu'elle l'est pour $37,6 \%$ des médicaments traditionnels de Niamey.

Cette durée de conservation des médicaments issus de différentes recettes n'est pas déterminée pour 384 recettes (Tableau III).

Tableau III. Répartition des recettes en fonction de l'opération, du mode d'administration, et de la durée de conservation des médicaments traditionnels

\begin{tabular}{|c|c|c|c|c|c|c|}
\hline \multirow{2}{*}{ Variable } & \multicolumn{2}{|c|}{ Niamey } & \multicolumn{2}{c|}{ Tillabéri } & \multicolumn{2}{c|}{ Total } \\
\cline { 2 - 7 } & Effectif & $\%$ & Effectif & $\%$ & Effectif & $\%$ \\
\hline \multicolumn{7}{|c|}{ Opération } \\
\hline Aucune & 1 & 0,9 & 29 & 3,6 & 30 & 3,3 \\
\hline Carbonisation/Friture & 4 & 3,4 & 13 & 1,6 & 16 & 1,7 \\
\hline Décoction/Infusion & 57 & 48,7 & 321 & 40,4 & 379 & 41,5 \\
\hline
\end{tabular}




\begin{tabular}{|c|c|c|c|c|c|c|}
\hline Macération & 11 & 9,4 & 132 & 16,6 & 143 & 15,7 \\
\hline Pulvérisation & 40 & 34,2 & 249 & 31,3 & 289 & 31,7 \\
\hline Ecrasement & 4 & 3,4 & 52 & 6,5 & 56 & 6,1 \\
\hline Total & 117 & 100 & 796 & 100 & 913 & 100 \\
\hline \multicolumn{7}{|c|}{ Mode d'administration } \\
\hline Absorption & 88 & 75,21 & 615 & 77,3 & 708 & 77,6 \\
\hline Application & 19 & 16,24 & 126 & 15,8 & 140 & 15,3 \\
\hline $\begin{array}{l}\text { Absorption- } \\
\text { Application }\end{array}$ & 7 & 6 & 27 & 3,4 & 34 & 3,7 \\
\hline Autre & 3 & 2,6 & 28 & 3,5 & 31 & 3,4 \\
\hline Total & 117 & 100 & 796 & 100 & 913 & 100 \\
\hline \multicolumn{7}{|c|}{ Durée de conservation } \\
\hline Non conservable & 2 & 1,7 & 53 & 6,6 & 55 & 6 \\
\hline Jusqu'à 1 mois & 36 & 30,8 & 346 & 43,5 & 381 & 41,7 \\
\hline Au-delà d'un mois & 6 & 5,1 & 86 & 10,8 & 93 & 10,2 \\
\hline Non déterminé & 73 & 62,4 & 311 & 39,1 & 384 & 42,1 \\
\hline Total & 117 & 100 & 796 & 100 & 913 & 100 \\
\hline
\end{tabular}

\section{Espèces médicinales recensées}

La liste des espèces constituant les recettes de médicaments dans les deux régions, les noms des genres et familles ainsi que les organes utilisés, les indications en thérapeutique traditionnelle et la fréquence des plantes sont données par les Tableaux IV(a) à IV(m). 
Tableau IV(c). Plantes et recettes médicinales recensées

\begin{tabular}{|c|c|c|c|c|c|}
\hline Famille & Binôme latin & Nom en langues locales & $\begin{array}{l}\text { Nombre de } \\
\text { recettes }\end{array}$ & Indications & Parties utilisées \\
\hline \multirow{6}{*}{$\begin{array}{l}\text { Asclepiadaceae } \\
\text { (suite) }\end{array}$} & Caralluma dalzieli N.E.Br. & Djotti(P), Tobaye barzou(Z) & 1 & Otite & Tige \\
\hline & Glossonema boveanum (Decne) & Taaringuitsa(H), Ganda kassi(Z) & 3 & Insuffisance de lactation, convulsion du paludisme, gonococcie & Plante entière \\
\hline & Calotropis procera (Ait.) R. Br. & Tounfafia $(\mathrm{H})$, Sagaye $(\mathrm{Z})$ & 6 & Céphalées, morsure de serpent, inflammation, anxiété, agitations & $\begin{array}{l}\text { Ecorce, racine, écorce } \\
\text { racine, fruits, latex }\end{array}$ \\
\hline & Pergularia daemia (Forssk) Chiov. & Fataka(Z), Huataka(H) & 1 & Anxiété, agitations & Feuilles \\
\hline & Solenostemma argel (Del.) Hayne. & $\begin{array}{l}\text { Agallacham }(\mathrm{H}), \text { Agallacham }(\mathrm{T}) \\
\text { Aghalgum }(\mathrm{A})\end{array}$ & 1 & Toux, manque d'appétit & Feuilles \\
\hline & $\begin{array}{lll}\begin{array}{l}\text { Leptadenia } \\
\text { Decne. }\end{array} & \text { pyrotechnica } & \text { (Forssk.) } \\
\end{array}$ & 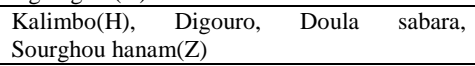 & 3 & Facilite le travail et l'accouchement, fièvre, paludisme & Plante entière \\
\hline Asparagaceae & Asparagus africanus Lam. & $\begin{array}{l}\begin{array}{l}\text { Foulan yollo, Garan } \\
\text { bahillata }(\mathrm{H})\end{array}\end{array}$ & 3 & Entretien des cheveux, douleurs gastriques & Feuilles, tige, plante entière \\
\hline \multirow[t]{5}{*}{ Asteraceae } & Centaurea perrottetii DC. & Kayan rakoumi $(\mathrm{H})$, Yo kargui $(\mathrm{Z})$ & 6 & $\begin{array}{l}\text { Conjonctivite, otite, drépanocytose, paludisme, fièvre, hématurie, douleurs } \\
\text { articulaires, hémorroïdes }\end{array}$ & $\begin{array}{llr}\begin{array}{l}\text { Feuilles, } \\
\text { entière }\end{array} & \text { fruits, plante }\end{array}$ \\
\hline & Vernonia amygdalina Delile & Shiwaka(H), Siwaka(Z) & 1 & Insuffisance de production lactée & Feuilles \\
\hline & Sonchus chevalieri & Kokulugna(Z), Ginagina(H) & 1 & Insuffisance de production lactée & Feuilles, tige \\
\hline & Dicoma tomentosa Cass. & Dawan makiaya $(\mathrm{H})$ & 1 & Lèpre & Plante entière \\
\hline & Acanthospermum hispidum DC. & Kashin yao(H) & 1 & Jaunisse, ulcère gastrique & Plante entière \\
\hline Balanitaceae & Balanites aegyptiaca $(\mathrm{L}$.$) Del.$ & Adoua(H), Tanni(P), Garbey(Z) & 25 & $\begin{array}{l}\text { Otite, plaie, piqûre de scorpion, panaris, hémorroïdes, asthénie, } \\
\text { inflammation, conjonctivite allergique, maux de ventre, constipation, } \\
\text { indigestion, dysentérie, jaunisse, troubles psychiques }\end{array}$ & $\begin{array}{l}\text { Feuilles, rameau, écorce, } \\
\text { racine, fruits }\end{array}$ \\
\hline \multirow[t]{2}{*}{ Bignoniaceae } & Stereospermum kunthianum Cham. & $\begin{array}{l}\text { Bollogna, Baritouri(Z) } \\
\text { Sansamé(H) }\end{array}$ & 4 & $\begin{array}{l}\text { Dysentérie chez la femme enceinte, jaunisse, asthénie, maux de ventre, } \\
\text { vertiges }\end{array}$ & Feuilles, écorce \\
\hline & Kigelia Africana (Lam.) Benth. & Kombay(Z), Yaourya(H) & 9 & Drépanocytose, diabète, plaie, plaie du diabétique, dysentérie, gastrite & Feuilles, écorce, fruits \\
\hline \multirow[t]{3}{*}{ Bombacaceae } & Adansonia digitata $\mathrm{L}$. & $\mathrm{Ko}(\mathrm{Z}), \mathrm{Kouka}(\mathrm{H})$ & 6 & $\begin{array}{l}\text { Constipation, parasitose intestinale, douleurs articulaires, hémorroïdes, } \\
\text { brulûre }\end{array}$ & Feuilles, écorce, fruits \\
\hline & Bombax costatum Pellegr. & Kurya(H), Forgo(Z) & 5 & $\begin{array}{l}\text { Ulcère gastrique, HTA, oedème, hémorroïdes, dysentérie, douleurs } \\
\text { abdominales }\end{array}$ & Feuilles, écorce, gomme \\
\hline & Ceiba pentandra (L.) Gaertn. & Bantan(Z), Rini(H) & 1 & Ulcère gastrique & Écorce \\
\hline Borraginaceae & Heliotropium ovalifolium Forssk. & Moyena(Z) & 1 & Maux de ventre & Feuilles \\
\hline
\end{tabular}

Tableau IV(d). Plantes et recettes médicinales recensées

\begin{tabular}{|c|c|c|c|c|c|}
\hline Famille & Binôme latin & Nom en langues locales & $\begin{array}{l}\text { Nombre de } \\
\text { recettes }\end{array}$ & Indications & Parties utilisées \\
\hline Burseraceae & $\begin{array}{l}\text { Commiphora Africana (A. Rich.) } \\
\text { Engl. }\end{array}$ & Iskitchi(H), Korombé(Z) & 7 & $\begin{array}{l}\text { Indigestion, nausées, vomissements douleurs abdominales, ulcère gastrique, } \\
\text { fièvre nocturne, asthénie, jaunisse, stérilité chez la femme }\end{array}$ & $\begin{array}{l}\text { Feuilles, rameau, écorce, } \\
\text { fruits, racine }\end{array}$ \\
\hline \multirow[t]{2}{*}{ Caesalpiniaceae } & Bauhinia rufescens Lam. & Jirga(H), Hay ga hampa, Namari(Z) & 16 & $\begin{array}{l}\text { Bonne vitalité chez l'enfant, apprentissage de la marche, diarrhée, douleurs } \\
\text { abdominales, angine, HTA, diabète, chute de cheveux, fatigue générale }\end{array}$ & $\begin{array}{l}\text { Feuilles, rameau, fruits, } \\
\text { racine }\end{array}$ \\
\hline & Cassia occidentalis $\mathrm{L}$. & Sangago, Sanga sanga, Sangata $(\mathrm{Z})$ & 19 & Paludisme, fièvre, maux de ventre, hémorroïdes, toux dysurie, jaunisse, & Feuilles, plante entière \\
\hline
\end{tabular}




\begin{tabular}{|c|c|c|c|c|c|}
\hline & & Kinkilba(H) & & œdème, douleurs lombaires & \\
\hline & Cassia sieberiana DC. & $\begin{array}{l}\text { Sinsan, Gougou(Z), Malga(H) } \\
\text { Sissangnahi(P) }\end{array}$ & 20 & $\begin{array}{l}\text { Énurésie, infection urinaire manque d'appétit, maux de ventre, indigestion, } \\
\text { hémorroïde, énuresie, fièvre, paludisme, douleurs cardiaques, douleurs } \\
\text { gastriques, drépanocytose, allergie cutanée }\end{array}$ & $\begin{array}{l}\text { Feuilles, écorce, racine, } \\
\text { écorce racine }\end{array}$ \\
\hline & Cassia italica (Mill.) F. W. Anders & Agargar(Z), Hilisko(H) & 15 & $\begin{array}{l}\text { Constipation chronique, facilite la digestion, maux de ventre, hémorroïdes } \\
\text { chroniques, fièvre typhoïde, drépanocytose, crise drépanocytaire }\end{array}$ & Feuilles, plante entière \\
\hline & Cassia mimosoides $\mathrm{L}$. & $\begin{array}{l}\text { Bagaroua kassa(H) } \\
\text { Ganda baani, dossari kirey }(\mathrm{Z})\end{array}$ & 2 & Douleurs abdominales, dysentérie, hémorroïdes, douleurs à la poitrine & Plante entière \\
\hline & Cassia nigricans Vahl. & $\begin{array}{l}\text { Niangalboubou(Z) Madatchin kassa, Gewaya } \\
\text { tsamia(H) }\end{array}$ & 10 & $\begin{array}{l}\text { agitation en cas de paludisme chez l'enfant, vertiges, jaunisse, maux de } \\
\text { ventre, indigestion, hémorroïdes, constipation, plaie, jaunisse }\end{array}$ & Feuilles, plante entière \\
\hline & $\begin{array}{lll}\text { Piliostigma } & \text { reticulatum } & \text { (DC.) } \\
\text { Hochst } & & \end{array}$ & Kossey, kossorey(Z), Kalgo(H) & 21 & $\begin{array}{l}\text { Soins après accouchement, bonne production de lait, fatigue, blessure, } \\
\text { circoncision, allergie cutanée, angine, hémorrö̈des, indigestion, maux de } \\
\text { ventre, dysentérie, douleurs cardiaques, jaunisse }\end{array}$ & Feuilles, écorce, rameau \\
\hline & Tamarindus indica $\mathrm{L}$. & Bossey(Z), Tsamia(H) & 7 & Constipation, asthénie, hémorroïdes, allergie & $\begin{array}{l}\text { Feuilles, écorce, racine, } \\
\text { fruits }\end{array}$ \\
\hline & $\begin{array}{l}\text { Detarium microcarpum Guill. et } \\
\text { Perr. }\end{array}$ & Fantou(Z), Taoura(H) & 1 & Ulcère gastrique & Écorce \\
\hline \multirow[t]{3}{*}{ Capparidaceae } & Boscia angustifolia A. Rich. & $\begin{array}{l}\text { Farin boka, Farin maouro, Farin moutoun, } \\
\text { Agagini }(\mathrm{H}), \text { Hassou koirey }(\mathrm{Z})\end{array}$ & 7 & $\begin{array}{l}\text { Agitations, anxiété, insomnies, convulsions, stérilité masculine, douleurs } \\
\text { rhumatismales }\end{array}$ & Ecorce \\
\hline & Boscia salicifolia Oliv. & Zougouré, Zouré(H), Yololo(Z) & 4 & Blessure, hoquet, insuffisance de production lactée & Feuille, rameau \\
\hline & Maerua angolensis DC. & $\begin{array}{l}\text { Koubou hotto, Balkou(Z), Miyahoua, } \\
\text { Tchitchiwa(H), Laguêhé, Helfittaye(P) }\end{array}$ & 3 & Parasitose intestinale, amaigrissement, panaris, plaie, hemorroïdes & Feuilles, tige \\
\hline
\end{tabular}

Tableau IV(e). Plantes et recettes médicinales recensées

\begin{tabular}{|c|c|c|c|c|c|}
\hline Famille & Binôme latin & Nom en langues locales & $\begin{array}{l}\text { Nombre de } \\
\text { recettes }\end{array}$ & Indications & Parties utilisées \\
\hline \multirow[t]{8}{*}{ Capparidaceae (suite) } & $\begin{array}{l}\text { Boscia senegalensis (Pers.) Lam ex } \\
\text { Poir }\end{array}$ & Anza(Z), Anza, Lolinhoua(H), Guiguili(P) & 14 & $\begin{array}{l}\text { Carie dentaire, inflammation, entorse, drépanocytose, inflammation, } \\
\text { maux de tête persistants, rhumatismes, hémorroïdes, agitations, } \\
\text { convulsions }\end{array}$ & $\begin{array}{l}\text { Feuilles, rameau, écorce, racine, } \\
\text { écorce racine, fruits }\end{array}$ \\
\hline & Cadaba glandulosa Forsk. & Atchou(P), Taïs(Z) & 3 & Epistaxis, maux de ventre & Feuilles \\
\hline & Cleome gynandra $\mathrm{L}$. & Gachia $(\mathrm{H})$, Houbey $(\mathrm{Z})$ & 5 & Otite, hémorroïdes & Feuilles \\
\hline & Maerua crassifolia Forsk. & Hassoubi $(\mathrm{Z})$, Jyga $(\mathrm{H})$ & 2 & $\begin{array}{l}\text { Parasitose intestinale, insuffisance de production lactée, diabète, } \\
\text { sinusite }\end{array}$ & Feuilles \\
\hline & Maerua angolensis DC. & $\begin{array}{l}\text { Koubou hotto, Balkou(Z), Miyahoua, } \\
\text { Tchitchiwa(H), Laguêhé, Helfittaye(P) }\end{array}$ & 3 & Parasitose intestinale, amaigrissement, panaris, plaie, hemorroïdes & Feuilles, tige \\
\hline & $\begin{array}{l}\text { Boscia senegalensis (Pers.) Lam ex } \\
\text { Poir }\end{array}$ & Anza(Z), Anza, Lolinhoua(H), Guiguili(P) & 14 & $\begin{array}{l}\text { Carie dentaire, inflammation, entorse, drépanocytose, inflammation, } \\
\text { maux de tête persistants, rhumatismes, hémorroïdes, agitations, } \\
\text { convulsions }\end{array}$ & $\begin{array}{l}\text { Feuilles, rameau, écorce, racine, } \\
\text { écorce racine, fruits }\end{array}$ \\
\hline & Cadaba farinosa Auct. & Bagaye $(\mathrm{H})$, Bagaye $(\mathrm{Z})$ & 1 & Parasitose intestinale & Feuilles \\
\hline & Corchorus olitorius $\mathrm{L}$. & Yao fokou (Z) & 1 & Fièvre typhoïde & Plante entière \\
\hline Caricaceae & Carica papaya $\mathrm{L}$. & Godda nassara $(\mathrm{H})$ & 6 & Jaunisse,fièvre, drépanocytose, hémorroïdes & Feuilles \\
\hline
\end{tabular}




\begin{tabular}{|c|c|c|c|c|c|}
\hline Caryophyllaceae & Polycarpea linearifolia DC. & Kansofoua $(\mathrm{H})$, Arkoussou bon koirai (Z) & 2 & Diarrhée chez l'enfant, ulcère gastrique & Plante entière \\
\hline Chrysobalanaceae & $\begin{array}{l}\begin{array}{l}\text { Neocarya macrophylla } \\
\text { Prance }\end{array} \\
\text { (Sabine) }\end{array}$ & Gamsa (H), Gamsa (Z) & 1 & Parasitose intestinale & Écorce \\
\hline Clusiaceae & Garcinia kola Heckel & Namijin goro(H), Goro arou(Z) & 1 & Douleurs cardiaques & Fruit \\
\hline Cochlospermaceae & Cochlospermum planchonii Hook F & Samaré (Z), Balagandé, Balgué (H) & 7 & Jaunisse, hémorroïdes & Racine, écorce racine \\
\hline \multirow[t]{4}{*}{ Combretaceae } & Combretum micranthum G. Don. & Koubou(Z), Guéza(H) & 26 & $\begin{array}{l}\text { Fatigue, nausées, vomissement, diarrhée, douleurs abdominales, } \\
\text { indigestion, ulcère gastrique, angine, douleurs lombaires, } \\
\text { courbatures, hémorroïdes, métrorragies, parasitose intestinale, plaie, } \\
\text { paludisme }\end{array}$ & Feuilles, rameau, écorce, racine \\
\hline & Guiera senegalensis J. G. Gmel. & Sabara(H), Sabara(Z), Djolooki(P) & 37 & $\begin{array}{l}\text { Allergie, conjonctivite, carie dentaire, vomissements diarrhée, } \\
\text { douleurs gastriques, maux de ventre, soins après accouchement, } \\
\text { fièvre, paludisme }\end{array}$ & $\begin{array}{l}\text { Feuilles, rameau, écorce, racine, } \\
\text { fruits }\end{array}$ \\
\hline & Pteleopsis suberosa Engl. et Diels & $\begin{array}{l}\text { Wouyan damo(H) } \\
\text { Yao barzou, Komni guindé( } \mathrm{Z})\end{array}$ & 4 & $\begin{array}{l}\text { Bon développement de l'enfant, diarrhée, indigestion, maux de } \\
\text { ventre }\end{array}$ & Ecorce \\
\hline & Combretum aculeatum Vent. & Boubouré(Z), Bubukia(H), Laougni(P) & 11 & Dysurie, paludisme, fièvre, hémorroïdes, maux de ventre & Feuilles, rameau, écorce, racine \\
\hline
\end{tabular}

\section{Tableau IV(f). Plantes et recettes médicinales recensées}

\begin{tabular}{|c|c|c|c|c|c|}
\hline Famille & Binôme latin & Nom en langues locales & $\begin{array}{l}\text { Nombre } \\
\text { de } \\
\text { recettes }\end{array}$ & Indications & Parties utilisées \\
\hline \multirow[t]{5}{*}{$\begin{array}{l}\text { Combretaceae } \\
\text { (suite) }\end{array}$} & $\begin{array}{l}\text { Anogeissus leiocarpus (DC.) } \\
\text { Guill. et Perr. }\end{array}$ & Mareké(H), Gonga(Z), Kodjioli(P) & 18 & $\begin{array}{l}\text { Jaunisse, allergie, plaie, paludisme, hémorroïdes, rhume, } \\
\text { asthme, parasitoses intestinales }\end{array}$ & Feuilles, rameau, écorce, racine \\
\hline & Combretum paniculatum Vent. & Zawana(H) & 1 & anémie & Feuilles, rameau \\
\hline & $\begin{array}{l}\text { Combretum glutinosum Perr. ex } \\
\text { DC. }\end{array}$ & Kokorbé(Z), Taramnia(H) & 18 & $\begin{array}{l}\text { Douleurs cardiaques, cancer du sein, carie dentaire, } \\
\text { vomissements chez l'enfant, menace d'avortement, troubles } \\
\text { digestifs, hémorroïdes, jaunisse }\end{array}$ & Feuilles, écorce, gomme \\
\hline & $\begin{array}{l}\text { Terminalia avicennioides Guill. } \\
\text { Et Perr. }\end{array}$ & $\begin{array}{l}\text { Karey hanga, Farka hanga(Z) } \\
\text { Bawshi(H) }\end{array}$ & 6 & Hémorroïdes, jaunisse, asthme & Feuilles, rameau, écorce, racine \\
\hline & Terminalia mantaly H. Perr. & & 1 & Hémorroïdes & Feuilles \\
\hline Commelinaceae & Commelina forskalei Vahl. & Balassa(H), Balassa(Z) & 1 & Déshydratation du nourrisson & Feuilles \\
\hline \multirow[t]{4}{*}{ Convolvulaceae } & Ipomoea aitonii Lindl. & Tchan hanga(Z), Kounnan kûssa(H) & 1 & HTA, facilite l'apprentissage de la marche & Plante entière \\
\hline & $\begin{array}{l}\text { Ipomoea asarifolia (Desr.) Roem. } \\
\text { Et Schult. }\end{array}$ & Talhana(Z), Douman kada(H) & 11 & $\begin{array}{l}\text { Drépanocytose, rhumatisme, douleurs articulaires, allergie } \\
\text { cutanée, chute de cheveux, hémorroïdes }\end{array}$ & Feuilles, tige, plante entière \\
\hline & $\begin{array}{l}\text { Merremia tridentate (L.) Hallier } \\
\text { F. }\end{array}$ & Yampourourou(H), Kognay zara(Z) & 1 & jaunisse & Plante entière \\
\hline & Evolvulus alsinioides $\mathrm{L}$. & $\begin{array}{l}\text { Moussou mé hamni, Souda fiti (Z), } \\
\text { Kafimalan fari, Kahi boka(H) }\end{array}$ & 3 & chute de cheveux, rhumatisme, hémorroïdes & Plante entière \\
\hline Cucurbitaceae & Momordica balsamina $\mathrm{L}$. & $\begin{array}{l}\text { Badoma }(\mathrm{Z}), \\
\text { Kolambayniya(H) }\end{array}$ & 18 & $\begin{array}{l}\text { Dermatose infectieuse, gale, fièvre, parasitose intestinale, } \\
\text { conjonctivite, fatigue, diarrhée, jaunisse, hémorroïdes }\end{array}$ & Feuilles, tige, plante entière \\
\hline
\end{tabular}




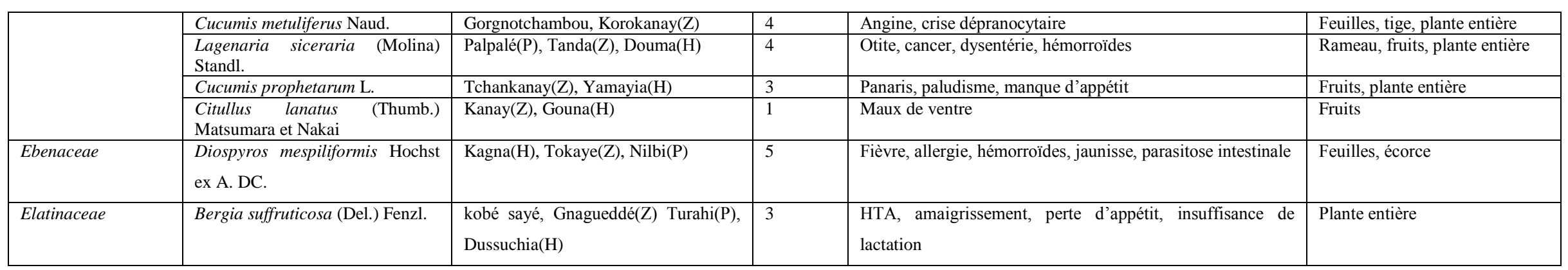

\section{Tableau IV(g). Plantes et recettes médicinales recensées}

\begin{tabular}{|c|c|c|c|c|}
\hline Famille & Binôme latin & Nom en langues locales & $\begin{array}{l}\text { Nombre } \\
\text { de } \\
\text { recettes }\end{array}$ & Indications \\
\hline Elatinaceae (suite) & Neptunia oleracea Lour. & $\begin{array}{l}\text { Tabataba }(\mathrm{Z}) \text {, Amakarékal(T) } \\
\text { Tataré tchina ka matan sarki sou } \\
\text { houtché(H) }\end{array}$ & 4 & $\begin{array}{l}\text { Fortifiant pour les enfants, maux de ventre chez les enfants, } \\
\text { otite, panaris }\end{array}$ \\
\hline \multirow[t]{10}{*}{ Euphorbiaceae } & Chrozophora brocchiana Vis. & Doré(Z), Damaigui, Bissa(H) & 12 & $\begin{array}{l}\text { Parasitose intestinale, diarrhée, douleurs abdominales, } \\
\text { dysentérie, démangeaisons, carie dentaire, hémorroïdes, } \\
\text { manque d'appétit }\end{array}$ \\
\hline & $\begin{array}{l}\text { Phyllanthus pentandrus Schum et } \\
\text { Tonn. }\end{array}$ & $\begin{array}{l}\text { Kolgney haïni(Z), Gaurihi(P) } \\
\text { Hatsin kourtia(H) }\end{array}$ & 4 & $\begin{array}{l}\text { Sevrage de l'enfant, piqûre de scorpion, maux de ventre, } \\
\text { indigestion }\end{array}$ \\
\hline & Jatropha curcas $\mathrm{L}$. & $\operatorname{Kidi}(\mathrm{P})$ & 2 & Maux de tête persistants, céphalées \\
\hline & $\begin{array}{l}\text { Flueggea virosa (Rxb. ex Wild) } \\
\text { Voigt }\end{array}$ & Sankana(Z), Tsa(H), Silmouyi(P) & 12 & $\begin{array}{l}\text { Dermatose allergique, dermatose infectieuse, drépanocytose, } \\
\text { maux de ventre, douleurs cardiaques }\end{array}$ \\
\hline & Euphorbia soudanica A Chev. & Mourtsoungoi(H), Dena bassou(Z) & 1 & Dermatose infectieuse \\
\hline & Euphorbia balsamifera Ait. & Barré(Z), Agoua(H) & 7 & $\begin{array}{l}\text { Planification familiale, maux de ventre, insuffisance de } \\
\text { lactation, oedèmes inflammatoires, gonococcie, infection } \\
\text { urinaire, hémorroïdes, douleurs gastriques }\end{array}$ \\
\hline & Ricinus communis L. & Zourma(H) & 1 & gonococcie \\
\hline & Tabiban & Tabiban & 2 & blessure de la peau, aphtes, gingivites, plaie rebelle \\
\hline & Croton zambezicus Mull. Arg. & Koriba(H), Tondi bon haïni $(\mathrm{Z})$ & 2 & Constipation chronique, ulcère d'estomac, crise drépanocytaire \\
\hline & Euphorbia hirta L. & Kolgneywa(Z), Nonon kourtchia(H) & 2 & Gonococcie, douleurs gastriques \\
\hline Fabaceae & Indigofera tinctoria $\mathrm{L}$. & Sini(Z), Baaba(H) & 4 & Métrorragies, carie dentaire, hémorroïdes \\
\hline
\end{tabular}

Parties utilisées

Feuilles, plante entière

Feuilles, plante entière

Plante entière, racine

Feuilles, rameau

Feuilles, rameau, écorce

Feuilles, rameau, écorce, latex

Feuilles, fruits, latex

Racine

Feuilles

Feuilles

Feuilles, racine

Plante entière

Plante entière, racine 


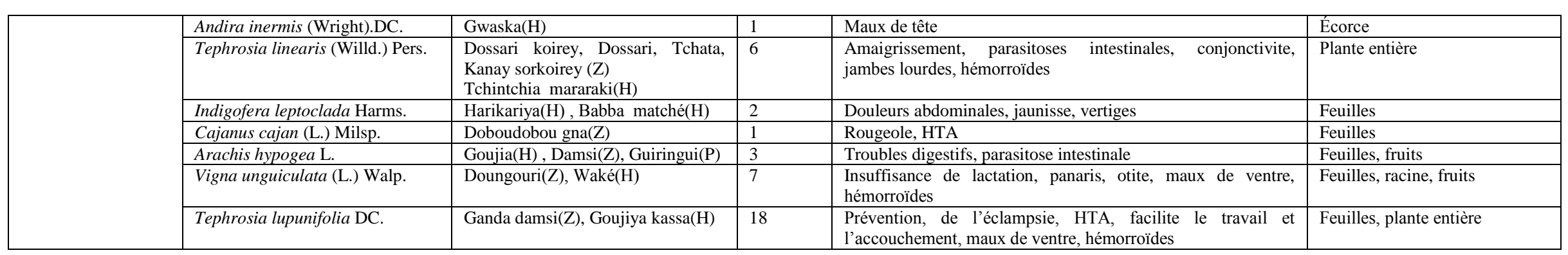

\section{Tableau IV(h). Plantes et recettes médicinales recensées}

\begin{tabular}{|c|c|c|c|c|c|}
\hline Famille & Binôme latin & Nom en langues locales & $\begin{array}{l}\text { Nombre } \\
\text { de } \\
\text { recettes }\end{array}$ & Indications & Parties utilisées \\
\hline \multirow{11}{*}{ Fabaceae (suite) } & Crotalaria arenaria Benth. & Tantaroba $(\mathrm{H})$, Tontonroba $(\mathrm{Z})$ & 2 & Manque d'appétit & Plante entière \\
\hline & $\begin{array}{l}\text { Tephrosia obcordata (Lam. Ex } \\
\text { Poir.) Backer. }\end{array}$ & Farka garbé(Z) & 2 & Dysentérie, hémorroïdes & Plante entière \\
\hline & Stylosanthes erecta P. Beauv. & $\begin{array}{l}\text { Kassan touri, Gangani fara, } \\
\text { Guidanbonsé(Z), Tsirahoko(H) }\end{array}$ & 6 & $\begin{array}{l}\begin{array}{l}\text { Fortifiant pour les enfants, rhume, troubles digestifs, } \\
\text { hémorroïdes }\end{array} \\
\end{array}$ & Feuilles, tige, plante entière \\
\hline & Crotalaria pallida Ait. & $\begin{array}{l}\text { Waynagana }(\mathrm{Z}) \text {, Fara birana }(\mathrm{H}) \text {, } \\
\text { Guini, Tinnam }(\mathrm{P})\end{array}$ & 4 & Fièvre, douleurs gastriques, douleurs abdominales & Feuilles, plante entière \\
\hline & Indigofera berhautiana Gillet. & $\begin{array}{lrrr}\text { Fégui mani, } & \text { Gaou } & \text { dambou, } \\
\text { Ganda bossey } & (\mathrm{Z}) & \text { Tsamia } \\
\text { kassa(H) } & & & \\
\end{array}$ & 3 & Manque d'appétit, amaigrissement, vertiges & Plante entière \\
\hline & Indigofera astragalina $\mathrm{DC}$. & $\begin{array}{l}\text { Soudji koukou(Z) } \\
\text { Kai kai koma kan machékia(H) }\end{array}$ & 4 & Infections ORL (angine, otite, aphtes, plaies de la bouche) & Feuilles \\
\hline & Pterocarpus erinaceus Poir. & Madobia(H) Tolo(Z) & 4 & Cancer, hémorroïdes & Rameau, écorce \\
\hline & Sesbania pachycarpa DC. & Kyerno $(\mathrm{H})$ & 1 & Jaunisse & Feuilles \\
\hline & $\begin{array}{l}\text { Zornia glauchidiata Reichb. Ex } \\
\text { DC. }\end{array}$ & Danguéro, Margou(Z) & 1 & Paludisme & Plante entière \\
\hline & Indigofera hirsuta $\mathrm{L}$. & Hakorin doki(H), Bari hingui(Z) & 1 & Maux de ventre & Plante entière \\
\hline & Abrus precatorius $\mathrm{L}$. & Zaaki(H) & 1 & Amaigrissement & Feuilles, plante entière \\
\hline \multirow[t]{2}{*}{ Flacourtiaceae } & Oncoba spinosa Forsk. & Tchibra $(\mathrm{H})$ & 1 & Jaunisse & Écorce \\
\hline & & Gargassa & 1 & $\begin{array}{l}\text { Saignements après accouchement, évacuer caillots de sang de } \\
\text { l'utérus après accouchement }\end{array}$ & Plante entière \\
\hline Hippocrateaceae & Loeseneriella africana (Willd.) & Dali(Z), Goday(H) & 1 & HTA, allergie & Feuilles \\
\hline
\end{tabular}




\begin{tabular}{|c|c|c|c|c|c|}
\hline & Wilczek & & & & \\
\hline Hyacinthaceae & $\begin{array}{l}\text { Dipcadi taccazeanum Hochst ex } \\
\text { A.Rich. }\end{array}$ & Gâdil(Z) & 1 & Stérilité & Bulbe \\
\hline kahi agougou & & kahi agougou $(\mathrm{H})$ & 1 & Parasitoses intestinales & Fruits \\
\hline \multirow[t]{2}{*}{ Lamiaceae } & Hyptis spicigera Lam. & Bounsouroun fadama $(\mathrm{H})$ & 1 & Anxiété, agitations & Plante entière \\
\hline & Ocimum gratissimum $\mathrm{L}$. & $\begin{array}{l}\text { koré gabou(Z), Kirmouï(P) } \\
\text { Kahi amaria kamchi(H) }\end{array}$ & 2 & Angine, allergie & Feuilles, plante entière \\
\hline Lauraceae & Persea Americana Mill. & & 1 & Jaunisse & Feuilles, fruit \\
\hline Loganiaceae & Strychnos innocua Del. & Kili kolôhi(P), Kouloukoulou(Z) & 2 & Fièvre typhoide, jaunisse & Feuilles, écorce \\
\hline Loranthaceae & Tapinanthus sp. A. Rich. & Kaoutchin sabara(H) & 1 & Énuresie & Plante entière \\
\hline Lythraceae & Lawsonia inermis $\mathrm{L}$. & Lallé(H), Alhinna(Z) & 2 & Stérilité masculine, drépanocytose & Feuilles \\
\hline
\end{tabular}

Tableau IV(i). Plantes et recettes médicinales recensées

\begin{tabular}{|c|c|c|c|c|c|}
\hline Famille & Binôme latin & Nom en langues locales & $\begin{array}{l}\text { Nombre } \\
\text { de } \\
\text { recettes }\end{array}$ & Indications & Parties utilisées \\
\hline \multirow[t]{4}{*}{ Malvaceae } & $\begin{array}{l}\text { Abutilon pannosum (Forsk.F.) } \\
\text { Schelchtend. }\end{array}$ & $\begin{array}{l}\text { Sarkin bissa, } \\
\text { Koira tombo(Z) }\end{array}$ & 9 & $\begin{array}{l}\text { Bilharziose, dysurie, gonococcie, infection urinaire, facilite le } \\
\text { travail et l'accouchement, allergie, déshydratation du } \\
\text { nourrisson }\end{array}$ & $\begin{array}{l}\text { Feuilles, écorce tige, racine, } \\
\text { écorce racine, plante entière }\end{array}$ \\
\hline & Hibiscus sabdariffa $\mathrm{L}$. & $\begin{array}{l}\text { Yakoua }(\mathrm{H}) \\
\text { Guissima, Waraou kirey }(\mathrm{Z})\end{array}$ & 3 & Diarrhée de la dentition, anémie, rhume & Fleurs \\
\hline & Gossypium barbadense mattei. & $\begin{array}{l}\text { Habou }(\mathrm{Z}) \\
\operatorname{Kadda}(\mathrm{H})\end{array}$ & 1 & Laxatif du nouveau-né & Feuilles, tige, fruits \\
\hline & Sida ovata Forsk. & Bari foy Kongaria(Z) & 2 & Blessure & Feuilles, plante entière \\
\hline \multirow[t]{3}{*}{ Meliaceae } & Azadirachta indica A. Juss. & $\begin{array}{l}\text { Milli, Dogon yaro }(\mathrm{H}) \\
\text { Milyayi(P), Millia(Z) }\end{array}$ & 20 & $\begin{array}{l}\text { Maux de ventre, hémorroïdes, paludisme, dysentérie, fièvre, } \\
\text { gastrite }\end{array}$ & Feuilles, écorce, racine, fruits \\
\hline & $\begin{array}{l}\text { Khaya senegalensis (Des.) A. } \\
\text { Juss. }\end{array}$ & Madatchi(H), Farrey(Z) & 18 & $\begin{array}{l}\text { Carie dentaire, diabète, maux de ventre, diarrhée, hémorroïdes, } \\
\text { douleurs articulaires, fatigue générale }\end{array}$ & Feuilles, écorce \\
\hline & $\begin{array}{l}\text { Pseudocedrela } \quad k o t s c h y i \\
\text { (Schweinf.)Harms. }\end{array}$ & Touna(H) & 3 & Dysentérie, rhumatisme & Feuilles, écorce \\
\hline Menispermaceae & $\begin{array}{l}\text { Chasmanthera dependens } \\
\text { Hochst }\end{array}$ & $\begin{array}{l}\text { Bouktey }(\mathrm{Z}) \\
\text { Douman doutsi }(\mathrm{H})\end{array}$ & 2 & Douleurs abdominales & Racine \\
\hline \multirow{3}{*}{ Mimosaceae } & Amblygonocarpus andongensis & Tsagué(H) & 1 & Facilite le sevrage de l'enfant & Ecorce \\
\hline & Acacia nilotica $\mathrm{L}$. & $\begin{array}{l}\text { Bilsa, Baani, Guitti(Z) } \\
\text { Bagaroua(H), } \\
\text { Gaoudi, Gawari(P) }\end{array}$ & 41 & $\begin{array}{l}\text { Toux, rhume, angine, aphtes, dysentérie, plaie rebelle, blessure } \\
\text { saignante, ulcère gastrique, infection urinaire, hémorroïdes, } \\
\text { colique, vers intestinaux, asthme, tuberculose pulmonaire, } \\
\text { diarrhée }\end{array}$ & Feuilles, écorce, racine, fruits \\
\hline & Acacia Senegal (L.) Willd. & Akora(H), Dangna(Z) & 2 & Furoncle, plaie & Racine \\
\hline
\end{tabular}




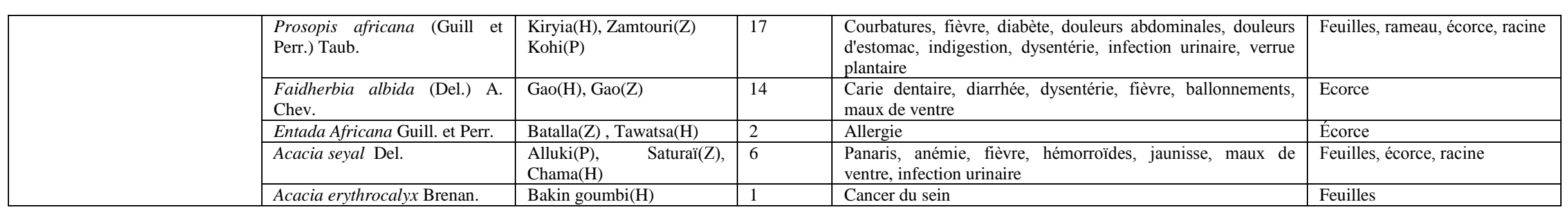

\section{Tableau IV(j). Plantes et recettes médicinales recensées}

\begin{tabular}{|c|c|c|c|c|c|}
\hline Famille & Binôme latin & Nom en langues locales & $\begin{array}{l}\text { Nombre } \\
\text { de } \\
\text { recettes }\end{array}$ & Indications & Parties utilisées \\
\hline \multirow[t]{3}{*}{ Mimosaceae (suite) } & Albizzia chevalieri Harms. & $\begin{array}{l}\text { Katsari(H), Dialiyahi(P), } \\
\text { Sana }(\mathrm{Z})\end{array}$ & 7 & $\begin{array}{l}\text { Asthénie, vertiges, aphtes, gingivites, diarrhée, allergie, ulcère d'estomac, } \\
\text { hémorroïdes }\end{array}$ & Feuilles, écorce \\
\hline & Prosopis juliflora (Sw.) DC. & Makka baani(Z) & 2 & Otite, maux de ventre & Feuilles \\
\hline & Parkia biglobosa (Jacq.) Benth. & Loutou(Z), Dorowa(H) & 2 & Hémorroïdes & Ecorce \\
\hline \multirow[t]{5}{*}{ Moraceae } & Ficus platyphylla Del. & $\begin{array}{l}\text { Kobé(Z), Gamji(H), } \\
\text { Iquiahi(P) }\end{array}$ & 5 & $\begin{array}{l}\text { Fatigue générale, oedèmes chez la femme enceinte, agitations et } \\
\text { insomnies, hémorroïdes }\end{array}$ & Feuilles, écorce \\
\hline & Ficus polita Vahl. & Durumi(H) , Durumi(Z) & 1 & Conjonctivite & Feuilles \\
\hline & Ficus thonningii Blume. & $\begin{array}{l}\text { Sédia }(\mathrm{Z}), \\
\text { Thiédyia(H) }\end{array}$ & 3 & Jaunisse, asthénie & Feuilles, écorce \\
\hline & Ficus sycomorus $\mathrm{L}$. & $\begin{array}{l}\text { Gaï gaï, Poorey(Z), } \\
\text { Bawri(H) }\end{array}$ & 3 & $\begin{array}{l}\text { Teigne, mycoses du cuir chevelu, maux de ventre, crise drépanocytaire, } \\
\text { coubartures vomissements, }\end{array}$ & Ecorce, latex \\
\hline & Ficus sp. & Rima djogohi(bérra) & 1 & Stérilité chez la femme & Fruits \\
\hline Moringaceae & Moringa oleifera Lam. & $\begin{array}{l}\text { Tabano, } \\
\text { boundou(Z), El makka(H) }\end{array}$ & 12 & $\begin{array}{l}\text { HTA, diabète, conjonctivite, apport de vitamines, crise dépranocytaire, } \\
\text { hémorroïdes }\end{array}$ & $\begin{array}{l}\text { Feuilles, rameau, } \\
\text { écorce, racine, fruits }\end{array}$ \\
\hline \multirow[t]{2}{*}{ Myrtaceae } & Eucalyptus camaldulensis Dehn. & Touraré(H), Touraré(Z) & 6 & Rhume, bronchite, crise drépanocytaire, anémie & Feuilles \\
\hline & Psydium guajava $\mathrm{L}$. & Goyba $(\mathrm{H})$ & 3 & Paludisme, hémorroïdes & Feuilles \\
\hline Nympheaceae & Nymphea lotus $\mathrm{L}$. & Bololi $(\mathrm{Z}), \operatorname{Bado}(\mathrm{H})$ & & Blennorragie & Plante entière \\
\hline Olacaceae & Ximenia Americana $\mathrm{L}$. & $\begin{array}{l}\text { Tsada, Mararuwa }(\mathrm{H}) \text {, } \\
\text { Moray, Molan }(\mathrm{Z})\end{array}$ & 14 & $\begin{array}{l}\text { Dracunculose, courbatures, HTA, gonococcie, plaie, hémorroïdes, } \\
\text { douleur abdominales, indigestion, jaunisse }\end{array}$ & $\begin{array}{l}\text { Feuilles, rameau, } \\
\text { écorce, racine, fruits }\end{array}$ \\
\hline \multirow[t]{2}{*}{ Pedaliaceae } & Rogeria adenophylla J. Gay. & $\begin{array}{l}\text { Lôda, Sassaga }(\mathrm{H}) \text {, } \\
\text { Atakourma lamti(Z) }\end{array}$ & 2 & HTA, ulcère gastrique & Feuilles, tige, fruits \\
\hline & Sesamum alatum Thon. & Lamtin baréwa(H), Koro & 4 & Facilite le travail, fatigue générale, infection urinaire, hémorroïdes & Fleurs, fruits, plante \\
\hline
\end{tabular}




\begin{tabular}{|c|c|c|c|c|c|}
\hline & & $\begin{array}{l}\text { lamti, Hansi lamti, Hansi } \\
\text { foy(Z) }\end{array}$ & & & entière \\
\hline & Sesamum indicum $\mathrm{L}$. & Lamti(H), Lamti(Z) & 1 & Rougeole & Fruits \\
\hline \multirow[t]{2}{*}{ Piperaceae } & Piper nigrum L. & Yagi $(\mathrm{H})$ & 1 & Douleurs lombaires & Fruits \\
\hline & $\begin{array}{l}\text { Piper guineense Schumach. et } \\
\text { Thonn. }\end{array}$ & Kirinfi (Z) & 3 & Bronchite & Fruits \\
\hline Poaceae & $\begin{array}{l}\text { Pennisetum americanum } \\
\text { Leeke }\end{array}$ & Haïni (Z) Hatsi(H) & 21 & $\begin{array}{l}\text { Fièvre, paludisme, dysentérie, asthénie, douleurs lombaires, douleurs } \\
\text { d'estomac, infestation par les vers intestinaux, hémorroïdes, dysentérie, } \\
\text { jaunisse, conjonctivite allergique, crise drépanocytaire, panaris, coliques }\end{array}$ & Epi, Grain \\
\hline
\end{tabular}

\section{Tableau IV(k). Plantes et recettes médicinales recensées}

\begin{tabular}{|c|c|c|c|c|c|}
\hline Famille & Binôme latin & Nom en langues locales & $\begin{array}{l}\text { Nombre } \\
\text { de } \\
\text { recettes }\end{array}$ & Indications & Parties utilisées \\
\hline \multirow[t]{10}{*}{ Poaceae (suite) } & Cymbopogon giganteus Chiov. & Tsabré(H), Soufarré(Z) & 2 & $\begin{array}{l}\text { Rhume, insomnie, agitation chez l'enfant, douleurs articulaires, } \\
\text { HTA }\end{array}$ & Feuilles, tige, plante entière \\
\hline & $\begin{array}{l}\text { Cymbopogon schoenanthus (L.) } \\
\text { Spreng. }\end{array}$ & Kodouré(Z), Nobi(H) & 2 & Constipation, jaunisse, drépanocytose, hémorroïdes & Feuilles, tige, plante entière \\
\hline & Pennisetum pedicellatum Trin. & Borboto(Z), Kyasoi(H) & 9 & $\begin{array}{l}\text { Infection urinaire, troubles urinaires, constipation, } \\
\text { démangeaisons }\end{array}$ & Feuilles, tige, plante entière \\
\hline & Aristida sieberiana Trin. & Yanta(H) & 2 & Jaunisse, drépanocytose, hémorroïdes & Plante entière \\
\hline & $\begin{array}{l}\text { Echinochloa stagnina (Retz.) P. } \\
\text { Beauv. }\end{array}$ & Koundou(P), Bourgou(Z) & 1 & Jaunisse & Plante entière \\
\hline & Eragrostis tremula Steud. & Koulum(Z), Burburwa(H) & 2 & Troubles digestifs & Racines \\
\hline & Zea mays L. & Massara $(\mathrm{H})$ & 2 & Asthme, stérilité chez la femme & Tige, feuilles, épi \\
\hline & Cenchrus prieurii (Kunth.) Maire. & Karfoka $(\mathrm{H})$ & 1 & HTA & Plante entière \\
\hline & Sorghum bicolor (L.) Moench. & Dawa $(\mathrm{H})$, Hamo $(\mathrm{Z})$ & 1 & Anémie & Feuilles \\
\hline & $\begin{array}{l}\text { Cymbopogon citratus (DC.) } \\
\text { Staph. }\end{array}$ & & 1 & Rhume, hémorroïdes & Feuilles, tige, plante entière \\
\hline Polygalaceae & $\begin{array}{l}\text { Securidaca longipedonculata } \\
\text { Fres. }\end{array}$ & $\begin{array}{l}\text { Zata aalali } \quad(\mathrm{Z}), \\
\text { Warnagoungouna, } \\
\text { Sagna(H) }\end{array}$ & 11 & $\begin{array}{l}\text { Maux de tête, maux de tête chroniques, ulcère gastrique, } \\
\text { drépanocytose, douleurs à la poitrine }\end{array}$ & Feuilles, écorce, racine \\
\hline Pontederiaceae & $\begin{array}{l}\text { Eichornia crassipes (Mart.) } \\
\text { Solms. Laub. }\end{array}$ & Kalâlou & 1 & Brûlure & Feuilles \\
\hline Renonculaceae & Nigella sativa $\mathrm{L}$. & Habat saouda & 1 & Fièvre, constipation & Graines \\
\hline \multirow[t]{2}{*}{ Rhamnaceae } & Ziziphus mauritiana Lam. & Magaria (darey, djabhi) & 20 & $\begin{array}{l}\text { Douleurs abdominales, dysentérie, règles douloureuses, } \\
\text { panaris, plaie }\end{array}$ & $\begin{array}{l}\text { Feuilles, écorce, écorce racine, } \\
\text { racine }\end{array}$ \\
\hline & Ziziphus mucronata Willd. & Koro darey $(\mathrm{Z}), \quad$ Magaria & 2 & Douleurs cardiaques & Feuilles, fruits \\
\hline
\end{tabular}




\begin{tabular}{|c|c|c|c|c|c|}
\hline & & koura $(\mathrm{H})$ & & & \\
\hline \multirow[t]{3}{*}{ Rubiaceae } & $\begin{array}{ll}\text { Crossopteryx febrifuga } & \text { (G. } \\
\text { Don.) } & \\
\text { Benth. }\end{array}$ & $\begin{array}{l}\text { Hinkin margou, } \\
\text { Hinkinmourzou(Z), } \\
\text { Makarhou(H) }\end{array}$ & 4 & Allergie & Feuilles, rameau \\
\hline & Sarcocephalus latifolius (Smith. & $\begin{array}{l}\text { Tafachia(H), Lolo(Z) } \\
\text { Bruce }\end{array}$ & 3 & Brulûres à la miction, jaunisse, HTA, fièvre, paludisme & Racine, écorce racine \\
\hline & $\begin{array}{l}\text { Gardenia ternifolia Schum. et } \\
\text { Thonn. }\end{array}$ & Komdi(Z), Gaoudé(H) & 7 & $\begin{array}{l}\text { Douleurs en cas d'hernie ombilicale du nouveau-né, favorise la } \\
\text { croissance de l'enfant, carie dentaire, maux de ventre, } \\
\text { ballonnements }\end{array}$ & $\begin{array}{l}\text { Feuilles, écorce racine, racine, } \\
\text { fruits }\end{array}$ \\
\hline
\end{tabular}

\begin{tabular}{|c|c|c|c|c|c|}
\hline Famille & Binôme latin & Nom en langues locales & $\begin{array}{l}\text { Nombre } \\
\text { de } \\
\text { recettes }\end{array}$ & Indications & Parties utilisées \\
\hline \multirow[t]{5}{*}{ Rubiaceae (suite) } & Gardenia sokotensis Hutch. & $\begin{array}{l}\text { Tondi fara(H), Goguedéli, } \\
\text { Babassey }(\mathrm{Z}) \text { Tchipaga }(\mathrm{P})\end{array}$ & 14 & $\begin{array}{l}\text { Manque d'appétit chez l'enfant, paludisme, retard de la marche } \\
\text { chez l'enfant, courbatures, indigestion, diarrhée, hémorrö̈des, } \\
\text { fatigue générale }\end{array}$ & Feuilles, rameau, écorce \\
\hline & $\begin{array}{l}\text { Mitragyna inermis (Willd.) O. } \\
\text { Kuntze. }\end{array}$ & Kabé(Z), Djayé(H) & 10 & $\begin{array}{l}\text { Hémorroïdes, hypertension artérielle, drépanocytose, vers } \\
\text { intestinaux, maux de ventre }\end{array}$ & feuilles, écorce, racine \\
\hline & Feretia apodenthera Del. & Fifirgui(Z), Tiguéréhi(P) & 2 & Paludisme, hémorroïdes & Feuilles, écorce \\
\hline & Mitracarpus scaber Zucc. & Hinkinia kanguié(Z) & 2 & $\begin{array}{l}\text { Dartres, autres mycoses de la peau, infection urinaire chez la } \\
\text { femme }\end{array}$ & Feuilles, plante entière \\
\hline & Pavetta crassipes K. Schum. & Gaddou(H) & 1 & Bronchite, rhume du bébé & Feuilles \\
\hline Rutaceae & Citrus limon (L.) Burm.f. & Lemou(H), Lemou(Z) & 5 & Rhume, dessèchement et fissures des lèvres & Feuilles, fruit \\
\hline Salvadoraceae & Salvadora persica $\mathrm{L}$. & Firaou $(\mathrm{Z})$ & 5 & Maux de ventre, hémorroïdes, anémie, rhume & Feuille, rameau, racine, fruit \\
\hline Sapindaceae & Paulinia pinnata $\mathrm{L}$. & Kambégou $(Z)$ & 1 & Amaigrissement, portage de vers intestinaux & Feuilles, tige \\
\hline Sapotaceae & $\begin{array}{l}\text { Vitellaria paradoxa } \text { C. } \mathrm{F} . \\
\text { Gaertn. }\end{array}$ & $\begin{array}{l}\text { Boulanga(Z), Kaddé(H), } \\
\text { Karéhé(P) }\end{array}$ & 6 & Bronchite, rhume enfant, crise drépanocytaire, conjonctivite & Feuilles, fruits, écorce \\
\hline \multirow[t]{3}{*}{ Scrophulariaceae } & Striga hermontica (Del.) Benth. & Malli(H) & 9 & $\begin{array}{l}\text { Diabète, démangeaisons, crise drépanocytaire, fièvre typhoide, } \\
\text { maux de tête, maux de ventre }\end{array}$ & Feuilles, plante entière \\
\hline & Scoparia dulcis $\mathrm{L}$. & Kahi malan, Ruma fada(H) & 2 & Fièvre, paludisme, démangeaisons & Feuilles, tige, plante entière \\
\hline & $\begin{array}{l}\text { Bacopa crenata (P. Beauv.) } \\
\text { Hepper. }\end{array}$ & Bangou mey dorey $(\mathrm{Z})$ & 1 & Maux de ventre & Plante entière \\
\hline \multirow[t]{3}{*}{ Solanaceae } & Solanum incanum $\mathrm{L}$. & $\begin{array}{l}\text { Kouloukoulou, Haou moy } \\
(\mathrm{Z}), \text { Kokia, Boussa, Idon } \\
\text { sania }(\mathrm{H})\end{array}$ & 3 & Prévision d'une bonne lactation chez la femme enceinte, otite & Fruits \\
\hline & Nicotiana tabacum L. & Taba $(\mathrm{H})$, Taba $(\mathrm{Z})$ & 1 & Asthme & Feuilles \\
\hline & Capsicum frutescens $\mathrm{L}$. & Tonko $\quad(\mathrm{Z}), \quad$ Tonka, & 4 & Rétention du placentaire après accouchement & Racine, fruits \\
\hline
\end{tabular}




\begin{tabular}{|c|c|c|c|c|c|}
\hline & & Barkono $(\mathrm{H})$ & & & \\
\hline & Solanum lycopersicon $\mathrm{L}$. & Toumati(H), Tomati(Z) & 1 & Gastrites & Feuilles \\
\hline & Schwenkia americana $\mathrm{L}$. & Guitti(Z), Dandana(H) & 2 & HTA & Plante entière \\
\hline \multirow[t]{2}{*}{ Sterculiaceae } & Waltheria indica $\mathrm{L}$. & $\begin{array}{l}\text { Ninebassi(Z), } \quad \text { Kafafi, } \\
\text { Poppotéki(P), } \\
\text { Yankoufa(H) }\end{array}$ & 13 & $\begin{array}{l}\text { Brûlure ou blessure, métrorragies, épistaxis, dysentérie chez } \\
\text { l'enfant, constipation chez l'enfant, soins après accouchement }\end{array}$ & $\begin{array}{l}\text { Feuilles, écorce racine, racine, } \\
\text { plante entière }\end{array}$ \\
\hline & Sterculia setigera Del. & $\begin{array}{l}\text { Tarkoundako(Z), } \\
\text { Koukouki(H) }\end{array}$ & 4 & Hypertension artérielle, ulcère gastrique, hémorroïdes & Feuilles, écorce, racine \\
\hline
\end{tabular}

Tableau IV(m). Plantes et recettes médicinales recensées

\begin{tabular}{|c|c|c|c|c|c|}
\hline Famille & Binôme latin & Nom en langues locales & $\begin{array}{l}\text { Nombre } \\
\text { de } \\
\text { recettes }\end{array}$ & Indications & Parties utilisées \\
\hline \multirow[t]{2}{*}{ Tiliaceae } & Grewia bicolor Juss. & $\begin{array}{ll}\text { Dargaza, } & \text { Darza }(H), \\
\text { Tassa }(Z)\end{array}$ & 3 & Douleurs cardiaques, brûlure, hémorroïdes, dysentérie & Ecorce, tige \\
\hline & Grewia flavescens Juss. & $\begin{array}{l}\text { Sari bon béri(Z), } \\
\text { Kamamoa(H) }\end{array}$ & 1 & Carie dentaire & Feuilles \\
\hline Tribulaceae & Tribulus terrestris $\mathrm{L}$. & Ngourfo(Z), Tsaïdo(H) & 2 & Règles douloureuses & Plante entière \\
\hline Verbenaceae & Vitex doniana Sweet. & Dumnia(H), Boyi(Z) & 3 & Hémorroïdes & Feuilles, écorce, fruits \\
\hline \multirow[t]{2}{*}{ Vitaceae } & Cissus quadrangularis $\mathrm{L}$. & $\begin{array}{l}\text { Tchébéri téli, Tarkounda } \\
\text { téli }(\mathrm{Z}) \text {, } \\
\text { Hanjin guiwa }(\mathrm{H})\end{array}$ & 3 & Allergie cutanée & Tige \\
\hline & $\begin{array}{l}\text { Ampelocissus grantii (Bak.) } \\
\text { Planch. }\end{array}$ & $\begin{array}{l}\text { Komnitanda, Tandari }(\mathrm{Z}) \\
\text { Douma douma }(\mathrm{H})\end{array}$ & 8 & $\begin{array}{l}\text { Cancer, inflammation, allergie peau, œdèmes, maux de ventre, } \\
\text { constipation }\end{array}$ & $\begin{array}{l}\text { Plante entière, écorce rhizome, } \\
\text { rhizome }\end{array}$ \\
\hline Zingiberaceae & Zingiber officinale Roscoe & $\operatorname{Tchitta}(\mathrm{H}), \operatorname{Tchitta}(\mathrm{Z})$ & 6 & $\begin{array}{l}\text { Hémorroïdes, carie dentaire, anémie, rhume, fièvre, } \\
\text { constipation, asthme }\end{array}$ & Rhizome \\
\hline
\end{tabular}




\section{Discussion \\ TPS enquêtés}

Le taux de refus est très élevé à Niamey $(39,4 \%)$. D’une manière générale, en Afrique les connaissances sur la médecine traditionnelle sont précieusement gardées par leurs détenteurs. Ce savoir s'accompagne assez souvent de pratiques rituelles et de mystères, et reste encore assujetti au poids des traditions orales, secrètes, conservées au fil des générations (OMS, 2002; Aké-Assi, 2006).

La réticence très marquée chez les TPS consultés à Niamey, grand centre urbain, peut s'expliquer par leur préoccupation à s'assurer des moyens de subsistance en maintenant une certaine notoriété dans le domaine de la thérapeutique traditionnelle. En effet, les conditions de vie y sont de plus en plus difficiles à l'instar de plusieurs grandes villes des pays en développement (INS, 2012; Adjanohoun et al., 1980) lors de l'enquête ethnobotanique dans les villes de Niamey, Dosso et Maradi constatait que les TPS rencontrés son généralement ouverts au dialogue.

Les personnes de notre enquête ont affirmé être de religion

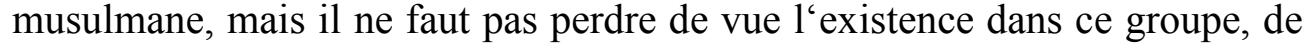
féticheurs, plus attachés aux coutumes ancestrales africaines, bien différentes de l'islam. Nacoulma (1996) dans son étude faite dans le Plateau central au Burkina Faso, a plutôt rencontré des TPS de confessions différentes (chrétiens, musulmans, féticheurs).

La prédominance des hommes $(85,71 \%$ dans l'ensemble, $80 \%$ à Niamey et $86,49 \%$ à Tillabéri), observée dans la population enquêtée témoigne de la première place qui leur revient dans l'exercice des professions au sein de nos sociétés, les femmes étant plutôt absorbées par les tâches ménagères. Apema et al (2010) dans ses travaux sur les plantes médicinales a trouvé $75 \%$ d'hommes parmi les TPS interrogés. Par contre Maiza et al. (1995) ont trouvé une prépondérance des femmes dans son échantillon, reflétant une différence de cultures entre les zones étudiées.

Plus de $90 \%$ des personnes interrogées ont un âge compris entre 25 et 75 ans dans les deux régions. C'est dans cette frange de la population qu'on retrouve la plupart des individus exerçant pleinement une profession. Aussi, l'activité de tradithérapeute est réservée aux personnes d'âge mûr. Pour être reconnu par sa communauté comme ayant des connaissances dans la médecine traditionnelle, il faut acquérir beaucoup de connaissances, ce qui exclut de cette profession les personnes les moins âgées $(0 \%$ à Niamey et $6,1 \%$ à Tillabéri). Maiza et al. (1995) ont également constaté que les informateurs de son enquête sont pour la plupart âgés.

Pour la majorité des personnes intérrogées de notre enquête $(82,73 \%)$ tout comme pour l'ensemble des TPS questionnés par Apema et al (2010), les connaissances sur la pharmacopée traditionnelle proviennent de la 
famille seulement, ce qui montre l'importance de la transmission filiale du savoir dans les mœurs africaines.

Plus de $60 \%$ de l'ensemble des TPS étudiés (102 soit 60,71\%) a exercé pendant au moins 10 ans. La durée d'exercice varie entre 2 et 75 ans ( 2 et 18 trouvés dans l'étude d'Apema); la moyenne de 25,76 ans, est plus élevée que celle constatée chez les personnes enquêtées à Bangui qui est de 12,5 ans (Apema et al., 2010).

Environ 20\% des TPS de notre travail ne situe pas dans le temps le début de l'activité en tant que tradipraticien, ce qui témoigne de leur faible niveau d'instruction.

L'activité principale est celle de tradipraticien pour la plupart des personnes enquêtées de la région de Niamey (85\%) alors qu'elle représente $33,11 \%$ à Tillabéri. La profession de TPS procure plus de revenus aux citadins, chez qui elle s'est développée aux dépens de l'agriculture et les autres professions qui ne représentent que $15 \%$, contre $66,89 \%$ dans la région de Tillabéri.

Aussi, l'agriculture qui constitue l'activité principale au Niger est presque abandonnée dans la capitale d'autant plus que ses revenus sont inconstants, car soumise aux aléas climatiques. Le manque de terres cultivables aux abords de la ville limiterait aussi la possibilité de pratiquer l'agriculture. Le milieu rural se prête aisément à cette activité comme le montre la place qu'elle occupe à Tillabéri $(47,3 \%)$.

\section{Recettes}

Les TPS font la consultation, le diagnostic et préparent le médicament comme décrit dans plusieurs travaux similaires réalisées en Afrique (Nacoulma, 1996).

Beaucoup de TPS de notre enquête ont déclaré être compétents dans le traitement de toutes les maladies. D'autres ne soignent qu'une catégorie d'affections à savoir les hémorroïdes (1 TPS), les maladies infantiles et féminines ( 9 matrones).

Même si les preuves scientifiques d'efficacité des médicaments traditionnels font défaut, ces produits bénéficient d'une longue expérience d'utilisation acquise depuis fort longtemps. Ils sont bien acceptés car la médecine traditionnelle est enracinée dans la vie socioculturelle des populations.

Le diagnostic souvent imprécis, n'est pas toujours évident, la méconnaissance de certaines maladies occasionne des biais dans la démarche, aboutissant à un traitement inadéquat (Bognon, 1991). C'est ainsi que nous avons écarté les données de 37 recettes relatives à un ensemble de symptômes regroupés sous le nom d'une affection appelée ''zahi' (en Haoussa) ou "wayno" (en Zarma). Cette maladie n'a pas de signification 
dans la médecine conventionnelle, et les signes décrits sont inconstants. La notion d'étiologie des maladies en médecine traditionnelle n'est pas toujours la même qu'en médecine moderne ainsi que l'interprétation des conceptions comme l'a constaté Bognon. A ce sujet, tant que le diagnostic n'a pas encore été élucidé, aucun rapprochement ni aucune intégration ne peuvent être envisagés entre la médecine traditionnelle et la médecine moderne, ce qui ne nous permet pas de les inclure dans notre étude. Cette clarification suppose une connaissance approfondie de la culture locale, condition essentielle pour comprendre le sens donné à certaines appellations. Le mode oral transmission des savoirs en Afrique, pourrait aussi avoir un impact sur l'explication des concepts (Wezel, 2002).

La durée de conservation des médicaments préparés à partir de différentes recettes est donnée pour 529 recettes sur les 913, soit (57,94\%). Les médicaments préparés ont un délai d'utilisation illimité dans 324 cas $(42,1 \%)$.

La notion de dose d'administration en fonction de l'âge du patient, nombre de doses journalières, moment d'administration, durée du traitement est différemment considérée dans le groupe étudié. La plupart des personnes de notre enquête (149 soit 88,7\%) n'ont que partiellement tenu compte de tous ces éléments de la posologie; 7 n'ont pas du tout abordé la posologie. Les termes employés lorsque cette posologie est absente ou incomplète sont entre autres " jusqu'à la guérison" pour la durée du traitement, auquel cas le médicament doit être administré pendant autant de jours que cela nécessite, ou 'à volonté", 'prendre une louche", 'prendre un pincée", 'un gobelet", "une tasse" etc..., dont le volume laisse à désirer, pour la quantité de médicament à donner. Ces informations sont entièrement déterminées dans toutes les recettes données par seulement 12 TPS.

Nous constatons que la thérapeutique traditionnelle possède des normes même si ces dernières ne sont pas suffisamment déterminées. Cela signifierait que la notion de normalisation du médicament et de sa posologie sont connues, mais leur importance mal discernée par les TPS. Le manque de dosage précis est un fait généralement reproché à la médication traditionnelle. De nos jours les tradithérapeutes donnent les directives concernant le dosage, mais elles peuvent être vagues (Sofowora, 1996). Les guérisseurs tentent de normaliser leurs préparations, mais cette standardisation est imprécise (Bongnon, 1991).

Selon Nacoulma (1996), un certain empirisme prévaut encore dans le domaine de la thérapeutique traditionnelle. L'efficacité thérapeutique et la posologie où la poignée, la pincée, la botte, la louche, la calebasse etc... comme unité de dosage, sont déterminées par tâtonnement, peu importe la quantité, le nombre de prises et la durée de la cure. 
Ngalamulume et al. (1995) affirmait que la médecine traditionnelle continue à rendre des services très appréciables dans beaucoup de pays en développement, mais elle laisse bien de gens insatisfaits du fait de l'imprécision qui y règne quant aux dosages et à la posologie. Cependant, la notion de toxicité existe chez les Peul du Sénégal oriental, selon lesquels il y a des plantes médicinales toxiques qui doivent être préparées avec soin (Van Der Steur, 1994).

Certaines recettes comptent plus d'une seule espèce végétale. Nous avons abandonné 6 recettes jugées complexes parce que comportant plus de 7 plantes. Dans ces recettes l'effet thérapeutique est peut être dû à une seule plante, qui est dissimulée par ajout d'autres espèces. La complexité des recettes pourrait traduire des besoins de secret, comme l'ont évoqué Nacoulma (1996) et Gangbo (1989).

Toutes les parties de la plante de même que la plante complète font l'objet de préparation de médicament avec une plus grande fréquence de l'appareil végétatif. Hassane (2008) a d'abord cité les feuilles, puis les fruits, les écorces, les racines, la plante entière ainsi que le latex ou la résine comme substance de base lors de la fabrication de médicaments traditionnels dans la réserve de Biosphère du $\mathrm{W}$ du Niger. La partie de plante utilisée est choisie en fonction de l'affection à traiter chez les TPS du Plateau central au Burkina Faso selon Nacoulma (1996), d'où l'emploi de plantes adultes ou leurs organes, mais aussi de germes de plantes, plantules, feuilles tombées, très tendres feuilles, drageons.

Les préparations de médicaments n'incorporent généralement pas d'autres substances $(91,9 \%)$. Divers produits biologiques ou minéraux sont associés dans les recettes de médicaments (champignon, escargot, natron, sel, etc....).

L'utilisation des champignons dans la pharmacopée traditionnelle de l'Afrique de l'ouest a aussi été signalée par Gerault et Thoen (1992).

L'emploi d'additifs animaux (os, fecès, gésier d'oiseau, animaux divers etc....) minéraux (argiles, natron, sel gemme, sel de potassium, etc....) dans la fabrication des médicaments a été décrit par d'autres auteurs (Nacoulma, 1996; Chifundera, 1992).

Le sucre n'est pas authentique à nos milieux, on peut attribuer son emploi dans les préparations aux habitudes de la vie citadine tout comme Nacoulma a supposé que l'utilisation du sucre est liée au modernisme.

La décoction et la pulvérisation représentent $72,61 \%$ des modes de préparation de médicaments dans l'ensemble des régions étudiées. Ces deux méthodes permettent une bonne conservation surtout dans le contexte du Niger où le climat chaud favorise la pourriture des produits organiques. La première est une ébullition de longue durée facilitant la destruction des 
microorganismes et la seconde une dessiccation empêchant leur multiplication.

Nacoulma (1996) a rapporté que la macération, la calcination, la carbonisation, la décoction, l'infusion, la lixiviation, la percolation, la pulvérisation, la torréfaction sont les principales opérations chez les tradithérapeutes de son étude.

Les médicaments sont le plus souvent absorbés (77,55\%), comme l'a indiqué Nacoulma (1996) dans son étude.

Les 226 espèces végétales appartiennent à 77 familles dont les plus représentées sont: Fabaceae (22 espèces), Euphorbiaceae (13 espèces), Mimosaceae (11 espèces), Poaceae (11 espèces), Combretaceae (10 espèces), Asclepiadaceae (9 espèces), Caesalpiniaceae ( 9 espèces), Capparidaceae (8 espèces), Rubiaceae ( 8 espèces), Anacardiaceae ( 6 espèces). L'importance de ces familles est à l'image de toute la flore du pays.

Notons qu'une même dénomination en langue locale peut désigner deux espèces différentes, ce qui fait que l'obtention de l'échantillon est capitale afin d'éviter les confusions, comme l'ont souligné Maiza et al. (1995).

Nos résultats corroborent ceux de Saadou (1993), Adjanohoun et al. (1980) où les Fabaceae (29 espèces) sont les plus importantes suivies de Caesalpiniaceae (14 espèces), Euphorbiaceae (12 espèces), Mimosaceae (11 espèces), Combretaceae (10 espèces), Capparidaceae (9 espèces), Poaceae (9 espèces).

Hassane (2008) a recensé pour les Fabaceae 22 espèces, les Mimosaceae 14 espèces, les Caesalpiniaceae 13 espèces, les Poaceae 10 espèces, Combretaceae 9 espèces, Euphorbiaceae 9 espèces, Rubiaceae 8 espèces, Capparidaceae 7 espèces comme familles les plus couramment utilisées en pharmacopée traditionnelle.

Thoen et Thiam (1990) dans une étude portant sur les espèces ligneuses et sub-ligneuses indigènes et exotiques qui existent dans la région du lac de Guiers, ont inventorié 131 espèces réparties en 49 familles parmi lesquelles les Mimosaceae (11 espèces), les Euphorbiaceae (11), les Capparaceae (9), les Caesalpiniaceae (9), sont les plus représentées.

Nous avons identifié 60 taxa qui s'ajoutent aux 245 déjà recensés comme espèces médicinales dans les travaux antérieurs réalisés et publiés au Niger. Saadou (1993) avait recensé 98 espèces nouvelles qui se sont ajoutées aux 147 espèces répertoriées par Adjanohoun et al. (1980).

Les échantillons de 8 taxa dont 4 n'ont pas été identifiés (faute de spécimen suffisant et en bon état de conservation) proviennent d'autres milieux et sont employés dans les recettes étudiées : alkafouna $(Z)$ ou tanouhouhou $(\mathrm{H})$, gargassa $(\mathrm{H})$, sipta $(\mathrm{Z})$, kahi agougou $(\mathrm{H})$, Amblygonocarpus andongensis, Piper nigrum, Piper guineense, Nigella 
sativa. Les recettes concernées par ces plantes sont probablement issues des pharmacopées des pays d'où proviennent les spécimens. Ceci reflète un certain brassage des cultures, occasionné par la proximité des peuples voisins et/ou le regroupement dans la capitale de personnes d'horizons divers. Nacoulma (1996) affirmait que les usages traditionnels des plantes sont nombreux, on assiste à des recoupements et croisements entre les recettes médicamenteuses.

D'après Saadou (1993), les familles qui présentent le plus grand nombre d'espèces nouvelles sont : les Fabaceae (9 espèces), Mimosaceae (7 espèces), Lamiaceae ( 6 espèces), Acanthaceae ( 6 espèces), Capparaceae ( 5 espèces), Cucurbitaceae ( 5 espèces) Poaceae ( 5 espèces).

Selon l'étude de Hassane (2008), les familles qui ont enregistré plus d'espèces nouvelles sont : Fabaceae (10 espèces), Cyperaceae (7 espèces), Malvaceae ( 5 espèces), Poaceae ( 5 espèces).

Dans notre lot de plantes nouvelles de la pharmacopée, les plus fréquemment utilisées sont : Alysicarpus ovalifolius (16 recettes), Eucalyptus camaldulensis (6), Allium sativum (6), Carica papaya (6), Citrus limon (5), Capsicum frutescens (4), Neptunia oleracea (4), Anacardium occidentale (3), Gymnema sylvestre (3), Glossonema boveanum (3), Sarcostemma vinimale (2), Strychnos innocu a (2).

Les espèces les plus fréquemment rencontrées dans l'ensemble des plantes recensées de notre étude sont: Sclerocarya birrea, Acacia nilotica, Guiera senegalensis, Annona senegalensis, Combretum micranthum, Balanites aegyptiaca, Pennisetum americanum, Piliostigma reticulatum, Cassia sieberiana, Ziziphus mauritiana, Azadirachta indica, Cassia occidentalis, Anogeissus leiocarpus, Combretum glutinosum, Khaya senegalensis, Momordica balsamina, Tephrosia lupunifolia, Prosopis africana, Alysicarpus ovalifolius, Bauhinia rufescens.

Ces espèces appartiennent aux familles suivantes: Anacardiaceae, Mimosaceae, Combretaceae, Annonaceae, Balanitaceae, Poaceae, Caesalpiniaceae, Rhamnaceae, Meliaceae, Cucurbitaceae, Fabaceae.

Certaines de ces plantes sont des plus citées comme espèces médicinales dans une étude réalisée par Modou et al. (1995); il s'agit de Guiera senegalensis, Cassia sieberiana, Cassia occidentalis, Adansonia digitata, Khaya senegalensis, Zizyphus mauritiana, Combretum micranthum et C. glutinosum.

Plusieurs plantes (16) de cet ensemble sont subspontanées. Ce sont: Allium sativum, Aloe vera, Carica papaya, Terminalia mantaly, Cajanus cajan, Arachis hypogea, Persea americanum, Prosopis juliflora, Eucalyptus camaldulensis, Zea mays, Cymbopogon citratus, Eichornia crassipes, Citrus limon, Capsicum frutescens, Solanum lycopersicon, Corchorus olitorius appartenant aux familles des Alliaceae, Aloaceae, Caricaceae, 
Combretaceae, Fabaceae, Lauraceae, Mimosaceae, Myrtaceae, Poaceae, Pontederiaceae, Rutaceae, Solanaceae, Tiliaceae.

Nous avons aussi trouvé $40(17,7 \%)$ espèces alimentaires qui sont : Allium sativum, Allium cepa, Mangifera indica, Xylopia aethiopica, Cocos nucifera, Vernonia amygdalina, Carica papaya, Manihot esculenta, Arachis hypogea, Vigna unguiculata, Persea americanum, Hibiscus sabdariffa, Moringa oleifera, Psydium guajava, Sesamum indicum, Piper guineense, Piper nigrum, Pennisetum americanum, Zea mays, Sorghum bicolor, Cymbopogon citratus, Citrus limon, Capsicum frutescens, Solanum lycopersicon, Corchorus olitorius, Zingiber officinale. Il y a donc des espèces médicinales $(82,3 \%)$, et des espèces à la fois alimentaires et médicinales $(17,7 \%)$.

L'espèce Guiera senegalensis qui est considérée comme envahissante pour avoir colonisé les plateaux dégradés de Djangoré (Mamadou, 2009) de plus en plus inadaptés aux cultures vivrières, s'est avérée très utile à la pharmacopée, car impliquée dans 37 recettes, avec des compétences médicale ou paramédicale. Ceci offre d'importantes opportunités d'exploitation au plan pharmaceutique.

Ces plantes inventoriées entrent dans la formulation des 913 recettes de produits traditionnels utilisés pour le traitement ou la prévention des maladies, mais aussi la modification des fonctions de l'organisme. Les concepts de prévention, de traitement ainsi que ceux de modification des fonctions organiques sont bien connus dans la thérapeutique traditionnelle. Les recettes destinées à prévenir les troubles de la dentition ou celles la planification des naissances en sont des exemples.

Les médicaments traditionnels répondent de ce fait à la définition du médicament donnée par le code de la santé publique (République du Niger, 1997).

Les recettes médicamenteuses traitent plusieurs affections, le plus souvent: troubles digestifs (hémorroïdes, maux de ventre, diarrhée, dysentérie, constipation, vomissements, indigestion, etc... : 364/913, soit $39,9 \%$ ), paludisme, fièvre, maux de tête $(74: 8,1 \%)$, pathologies des femmes et des enfants (69/913, soit 7,6\%), rhumatismes, inflammations (54/913), jaunisse (49/913), plaies diverses, infections de la peau (52/913), infections ORL, carie dentaire, infection oculaire (50/913), allergie (45/913), anémie, asthénie, manque d'appétit, parasitose (41/913), affections respiratoires (32/913), diabète (20/913), hypertension artérielle (19/913). D'après Hassane (2008), un grand nombre de plantes de son étude est utilisé pour traiter des maladies abdominales et des pathologies infantiles.

Les recettes relatives aux troubles digestifs sont généralement antihémorroïdaires (132/364, soit 36,3\%), antispasmodiques (102/364) antidiarrhéiques, antiamibiennes (54/234). Wezel (2002) constatait que les 
hémorroïdes étaient les pathologies les plus fréquemment soignées avec les médicaments traditionnels, suivis des maux de ventre, de la dysentérie. Selon l'étude de Hassane (2008), les maladies abdominales traitées sont principalement les hémorroïdes, la dysenterie, les maux de ventre, la diarrhée.

Il y a 83 recettes destinées aux pathologies des femmes enceintes ou allaitantes et des enfants, groupes appartenant aux couches les plus vulnérables de la société, et 46 d'entre elles consacrées aux enfants, dont 10 soit $21,7 \%$ sont antidiarrhéiques. D'après Hassane (2008), un grand nombre des plantes sont utilisées pour le traitement des maladies abdominales (hémorroïde, dysenterie, maux de ventre, diarrhée) et les pathologies infantiles.

L'usage para-médical (prévention des troubles de l'organisme et l'amélioration de son fonctionnement) concerne 39 recettes parmi lesquelles 32 sont employées pour les femmes et les enfants. Ceci montre l'attention portée en milieu traditionnel à cette importante frange de la population (INS, 2012).

Les recettes sont en général utilisées (757/913, soit 82,9\%) dans une seule situation (traitement, prévention etc....). Seules 156 préparations médicamenteuses (17\%) ont plus d'un emploi en thérapeutique traditionnelle.

Les plantes ont le plus souvent plusieurs utilisations (162/226 soit $71,7 \%$ ), au-delà de 8 pour les espèces suivantes: Annona senegalensis, Sclerocarya birrea, Allium sativum, Leptadenia hastata, Centaurea perrottetii, Balanites aegyptiaca, Commiphora africana, Bauhinia rufescens, Cassia occidentalis, Cassia sieberiana, Cassia nigricans, Piliostigma reticulatum, Boscia senegalensis, Combretum micranthum, Guiera senegalensis, Combretum glutinosum, Momordica balsamina, Euphorbia balsamifera, Acacia nilotica, Prosopis africana, Albizzia chevalieri, Ximenia americana, Pennisetum americanum, Gardenia sokotensis.

La plupart des plantes sont utilisées pour le traitement d'une à trois maladies selon l'enquête de Hassane (2008), au maximum 6 d'après celle de Saadou (1993), et plus de 8 usages pour la Pharmacopée d'Afrique de l'Ouest (2013).

\section{Conclusion}

Cette enquête nous a permis de constater que les régions de Niamey et Tillabéri renferment un riche éventail de plantes médicinales, avec 226 espèces dont 60 non précédemment rapportées et appartenant à 42 genres et 31 familles. Elles feront l'objet d'investigations complémentaires pour enrichir les monographies des plantes médicinales du Niger. Un autre constat relatif aux concepts de dose, nombre de doses quotidiennes, moments 
d'administration, durée du traitement, traitement curatif, traitement préventif, est fait dans notre pharmacopée traditionnelle comme dans celles des autres régions d'Afrique. Cependant, tous les effets thérapeutiques avancés des plantes ne sont pas attestés par des preuves scientifiques. Il n'y a pas eu suffisamment d'expérimentation pour distinguer les plantes réellement efficaces. Aussi, ces concepts (dosages, posologie) ne sont pas suffisamment précis car il n'y a pas eu de standardisation préalable.

D'importants défis sont à relever, il s'agit entre autres de:

continuer à inventorier toutes les plantes de notre pharmacopée ainsi que les recettes de médicaments traditionnels dans des zones peu explorées comme l'Aïr, l'Azawak, le Kawar;

- $\quad$ promouvoir une meilleure utilisation des plantes médicinales par la mise au point des médicaments traditionnels améliorés (MTA), après investigations phytochimiques voire pharmaco-toxicologiques;

- $\quad$ promouvoir la culture de certaines plantes médicinales menacées de disparition, et valoriser les plantes peu exigeantes ayant spontanément colonisé les zones dégradées du pays.

\section{References:}

1. Adjanohoun et al. (1980). Médecine et pharmacopée, Contribution aux études ethnobotaniques et floristiques au Niger. ACCT, $251 \mathrm{p}$.

2. Aké-Assi, L. (2006). Quelques plantes employées traditionnellement dans la couverture des soins de santé primaire. Abrégé de médecine et pharmacopées africaines, p. 9.

3. Akoègninou, A., van der Burg, WJ., van der Maesen, LJG., Adjakidjè, V., Essou, JP., Sinsin, B., Yédomonhan, H. (2006). Flore Analytique du Bénin. Backhuys Publishers, Wageningen.

4. Apema, R., Mozouloua, D., Abeye, J., Salamate, F. M. L. (2010). Plantes médicinales utilisées dans le traitement du diabète par les tradipraticiens à Bangui. Systématique et Conservation des Plantes Africaines. Comptes Rendus du 18ème Congrès de l'AETFAT, Yaoundé, Cameroun (du 11 au 12 fevrier 2010) : 297-303.

5. Berhaut, J. (1967). Flore du Sénégal. 2ème édition plus complète avec les forêts humides de la Casamance. Clairafrique Dakar, 485p.

6. Bognon, C. (1991). Notes ethnobotaniques sur la médecine traditionnelle en pays Wè (Côte d'Ivoire): quelques problèmes méthodologiques. Revue de Médecines et pharmacopées africaines, 5 (1) : 55-63.

7. Chifundera, K. (1992). Matières minérales et animales utilisées dans la médecine traditionnelle au Bushi, Sud-kivu, Est du Zaïre. Revue de Médecines et pharmacopées africaines 6 (1) : 33-44. 
8. Gangbo, Z. S. (1989). Contribution au processus de l'association des deux médecines moderne et traditionnelle : proposition de stratégie. Revue de Médecines et pharmacopées africaines, 3 (1) : 53-60.

9. Gerault, A., \& Thoen, D. (1992). Les champignons dans les pharmacopées traditionnelles de l'Afrique de l'Ouest. Revue de Médecines et pharmacopées africaines, 6 (1) : 45-53.

10. Hassane, H. (2008). Répertoire des espèces végétales les plus couramment utilisées en pharmacopée traditionnelle et impact des techniques de prélèvement sur la diversité biologique dans la réserve de Biosphère du W du Niger. Mémoire de DEA Géographie, Milieux et sociétés des espaces arides et semi-arides : AménagementDéveloppement. Université Abdou Moumouni de Niamey, 133 p.

11. Institut National de la Statistique (2012). Enquête démographique et de santé à indicateurs multiples. Niger, $145 \mathrm{p}$.

12. Maiza, K. H. et al. (1995). Pharmacopée traditionnelle saharienne. Revue de Médecines et pharmacopées africaines, 9 (1) : 71-77.

13. Mamadou, A. J. (2009). Analyse des gradients floristiques au sein des végétations de plateau selon un transect nord-sud dans la réserve de Biosphère. Mémoire de DEA Biologie Appliquée, Protection et Restauration des Ecosystèmes Soudaniens et Sahélo-sahariens, Université Abdou Moumouni de Niamey, 74p.

14. Modou, L. et al. (1995). Place des plantes médicinales dans le système de santé au Sénégal. Revue de Médecines et pharmacopées africaines 9 (1) : 43-47.

15. Nacoulma-Ouédraogo, O. G. (1996). Plantes médicinales et pratiques médicales traditionnelles au Burkina Faso, cas du Plateau central. Thèse de doctorat ès Sciences Naturelles, Université de Ouagadougou, 320 p. +262 p.

16. Ngalamulume, T. et al. (1995). Plantes médicinales à usage domestique cultivées dans deux quartiers de Kinshasa. Revue de Médecines et pharmacopées africaines, 9 (2) : 9-14.

17. OMS (2002). Stratégie pour la médecine traditionnelle 2002-2005. Genève.

18. Organisation Ouest Africaine de la Santé (2013). Pharmacopée d'Afrique de l'Ouest, $251 \mathrm{p}$.

19. Peyre De Fabregues, B. (1979). Lexique des plantes du Niger. IEMVPT, INRAN, $2^{\text {ème }}$ édition, $156 \mathrm{p}$.

20. République du Niger (1997). Législation pharmaceutique. MSP, Ordonnance $n^{\circ 97-002}$ du 10 janvier 1997.

21. Saadou, M. (1993). Les plantes médicinales du Niger: premier supplément à l'enquête ethnobotanique de 1979. Revue de Médecines et pharmacopées africaines, 7 (1) : 11-24. 
22. Saadou, M. (1998). Evaluation de la biodiversité biologique au Niger : éléments constitutifs de la biodiversité végétale. Conseil National de l'Environnement pour un Développement Durable SE/CNEDD, Projet NER/ 97 / G 31 / A / 1 G / 99 "Stratégie Nationale et plan d'action - Diversité Biologique", 138p.

23. Schmelzer, GH., Gurib-Fakim, A., Lemmens, RHMJ., Oyen Ipa Chauvet, M., Siemonsma, JS. (2008). Plantes Médicinales, Fondation PROTA/ Backhuys Publishers/CTA, Wageningen, Pays-Bas, p 869.

24. Sofowora, A. (1996). Plantes médicinales et médecine traditionnelle d'Afrique. Ed. Khartala, Paris, 256p.

25. Thoen, D., \& Thiam, A. (1990). Utilisations des plantes ligneuses et sub-ligneuses par les populations de la région sahélienne du lac de Guiers (sénégal). Revue de Médecines et pharmacopées africaines, 4 (2) : 169-177.

26. Van Der Steur, L. (1994). Plantes médicinales utilisées par les Peul du Sénégal oriental. Pharmacopée traditionnelle saharienne. Revue de Médecines et pharmacopées africaines, 8 (2) : 189-200.

27. Wezel, A. (2002). Plantes médicinales et leur utilisation traditionnelle chez les paysans du Niger. Etudes Flor. Vég. Burkina Faso, Frankfurt/ Ouagadougou, $6: 9-18$. 